\title{
From Subthreshold to Firing-Rate Resonance
}

\author{
Magnus J. E. Richardson, ${ }^{1,2}$ Nicolas Brunel, ${ }^{3}$ and Vincent Hakim ${ }^{\mathbf{1}}$ \\ ${ }^{1}$ Laboratoire de Physique Statistique, Ecole Normale Supérieure, 75231 Paris Cedex 05, France; ${ }^{2}$ Laboratory of Computational \\ Neuroscience, Brain and Mind Institute, Ecole Polytechnique Fédérale de Lausanne, CH 1015 Lausanne, Switzerland; and ${ }^{3}$ Centre National de \\ la Recherche Scientifique, Neurophysique et Physiologie du Système Moteur, Université René Descartes, 75270 Paris Cedex 06, France
}

Submitted 24 October 2002; accepted in final form 16 December 2002

Richardson, Magnus J. E., Nicolas Brunel, and Vincent Hakim. From subthreshold to firing-rate resonance. J Neurophysiol 89: 2538 2554, 2003. First published December 27, 2002; 10.1152/jn.00955. 2002. Many types of neurons exhibit subthreshold resonance. However, little is known about whether this frequency preference influences spike emission. Here, the link between subthreshold resonance and firing rate is examined in the framework of conductance-based models. A classification of the subthreshold properties of a general class of neurons is first provided. In particular, a class of neurons is identified in which the input impedance exhibits a suppression at a nonzero low frequency as well as a peak at higher frequency. The analysis is then extended to the effect of subthreshold resonance on the dynamics of the firing rate. The considered input current comprises a background noise term, mimicking the massive synaptic bombardment in vivo. Of interest is the modulatory effect an additional weak oscillating current has on the instantaneous firing rate. When the noise is weak and firing regular, the frequency most preferentially modulated is the firing rate itself. Conversely, when the noise is strong and firing irregular, the modulation is strongest at the subthreshold resonance frequency. These results are demonstrated for two specific conductance-based models and for a generalization of the integrate-and-fire model that captures subthreshold resonance. They suggest that resonant neurons are able to communicate their frequency preference to postsynaptic targets when the level of noise is comparable to that prevailing in vivo.

\section{IN T R O D U C T I O N}

Oscillations have long been observed in neuronal structures (Adrian and Matthews 1934) but their role, mechanisms, and interplay with single neuron biophysical characteristics have only recently been submitted to detailed scrutiny. Experiments have tested the response of neurons to oscillating current injection. Subthreshold resonance, in which the response of the induced oscillating voltage peaks at a preferred input frequency, has been found in inferior olive neurons (De Zeeuw et al. 1998; Lampl and Yarom 1993, 1997; Llinas and Yarom 1986), trigeminal root ganglion neurons (Puil et al. 1986), thalamic neurons (Hutcheon et al. 1994; Jahnsen and Karnup 1994; Puil et al. 1994), cortical neurons (Dickson et al. 2000; Gutfreund et al. 1995; Hutcheon et al. 1996b; Llinas et al. 1991), and both hippocampal CA1 pyramidal cells (Leung and Yu 1998; Pike et al. 2000) and interneurons (Pike et al. 2000). Many of these structures are known to support oscillations in vivo, suggesting an interplay between single-cell frequency

\footnotetext{
Address for reprint requests: M.J.E. Richardson, Laboratory of Computational Neuroscience, Brain and Mind Institute, EPFL, CH 1015, Lausanne, Switzerland (E-mail: Magnus.Richardson@epfl.ch).
}

preference and oscillations at the network level. Most of the recorded neurons show a single peak at a finite frequency in their voltage response. However, some interneurons of the hippocampus show a more complex response with a trough at low frequency followed by a peak at higher frequencies (Pike et al. 2000). Although a great deal of effort has been directed at understanding the input properties of resonant neurons, surprisingly little attention has been addressed to the effect of subthreshold resonance on the temporal properties of the firing rate. This is despite the common assumption that the presence of resonant neurons might provide a stabilizing influence on oscillations at the level of the network.

It is known from Hodgkin and Huxley (1952) and many studies since (see e.g., Gutfreund et al. 1995; Hutcheon et al. 1996a, 1994; Koch 1984; Mauro et al. 1970; Rinzel and Ermentrout 1989; White et al. 1995) that the resonance properties of neurons can be related to their ionic channel characteristics through a mathematical linearization of the corresponding conductance-based description. Several scenarios involving voltage-gated ionic currents have been shown to generate resonant behavior (for a review, see Hutcheon and Yarom 2000). Reduced two-variable descriptions have proven useful as a mathematical tool to study these and other neuronal properties (Gutfreund et al. 1995; Hutcheon et al. 1996a; Rinzel and Ermentrout 1989; White et al. 1995).

In the first part of this paper, a systematic classification of two-variable models is provided. The analysis highlights the possible types of subthreshold behavior associated with different neuronal characteristics. The results can be summarized in a graphical description. The change of membrane properties as the neuron is depolarized toward threshold is represented by trajectories crossing boundaries separating different types of behavior (e.g., passive from resonant). This description is illustrated with two conductance-based model neurons. More complex types of resonance cannot be described by a twovariable model. For this reason, a three-variable model, which exhibits a richer repertoire of behaviors, is also analyzed. A parameter region is identified with a suppression as well as a resonance in the impedance curve, a feature recently observed experimentally in hippocampal fast-spiking interneurons (Pike et al. 2000).

In the second part of the paper, the circumstances are examined in which a resonant neuron can communicate its sub-

\footnotetext{
The costs of publication of this article were defrayed in part by the payment of page charges. The article must therefore be hereby marked "advertisement" in accordance with 18 U.S.C. Section 1734 solely to indicate this fact.
} 
threshold frequency preference through the dynamics of its firing rate. This property of resonant neurons manifests itself as a preferential amplification of input signals that are at the resonant frequency and requires an analysis of how the firing rate is modulated by an oscillatory current. To this end, the two-variable approach is extended to include spike emission, providing a generalized integrate-and-fire or GIF model. The model captures a wide range of subthreshold dynamics with a simplified firing and reset mechanism. The firing-rate dynamics of this model, as well as two specific conductance-based models which exhibit a subthreshold resonance, are studied in detail. The crucial role that noise plays in shaping the response is highlighted.

\section{METHODS}

\section{Glossary}

$v \quad$ deviation of the membrane potential from the holding potential $(\mathrm{mV})$.

$C$ or $C_{M} \quad$ membrane capacity $(\mathrm{nF})$.

$g \quad$ effective leak conductance $(\mu \mathrm{S})$.

$w$ or $w_{1} \quad$ auxiliary variable characterizing the membrane dynamics $(\mathrm{mV})$.

$\tau_{1} \quad$ time scale of the dynamics of the $w$ variable (ms)

$g_{1} \quad$ conductance measuring the membrane potential variation resulting from a change of $w(\mu \mathrm{S})$.

$\alpha \quad$ dimensionless parameter proportional to leak conductance $g$.

$\beta$ dimensionless parameter proportional to conductance $g_{1}$.

$w_{2} \quad$ second auxiliary variable $(\mathrm{mV})$.

$g_{2} \quad$ analogous to $g_{1}$ for the second variable $w_{2}(\mu \mathrm{S})$.

$I_{\text {app }} \quad$ total applied external current (nA).

$I_{\text {syn }} \quad$ synaptic current (nA).

$g_{\text {eo }}$ and $g_{\text {io }}$ average excitatory and inhibitory total synaptic conductances $(\mu \mathrm{S})$.

$\sigma_{e}$ and $\sigma_{i} \quad$ magnitude of excitatory and inhibitory synaptic noise $(\mu \mathrm{S})$.

$\tau_{e}$ and $\tau_{i} \quad$ correlation timescales of the excitatory and inhibitory synaptic noise $(\mathrm{ms})$.

$I_{N} \quad$ magnitude of the fluctuations of synaptic current (nA).

$I_{0} \quad$ constant (DC) current (nA).

$I_{1} \quad$ magnitude of oscillatory current (nA).

$f \quad$ frequency of injected current $(\mathrm{Hz})$.

$\sigma_{V} \quad$ strength of the synaptic noise as measured by the resulting amplitude of membrane potential fluctuations $(\mathrm{mV})$.

$Z(f) \quad$ cell impedance for an injected current of frequency $f$ $(M \Omega)$.

$f_{R} \quad$ resonant frequency corresponding to a maximum of the amplitude of $Z(f)(\mathrm{Hz})$.

$f_{0} \quad$ natural frequency of the membrane potential damped oscillations $(\mathrm{Hz})$.

$Q \quad$ strength of the resonance peak (dimensionless).

$r_{0} \quad$ average spike rate $(\mathrm{Hz})$.

$r_{1}(f) \quad$ magnitude of oscillatory component in spike rate induced by injected oscillatory current $(\mathrm{Hz})$.

$|A(f)| \quad$ signal gain ( $\mathrm{Hz} / \mathrm{nA})$.

$\phi(f) \quad$ phase of oscillatory component in spike rate with respect to oscillatory current (deg).

$v_{\theta} \quad$ threshold for spike emission for the GIF model (defined in METHODS; $\mathrm{mV}$ )

$v_{r} \quad$ membrane potential reset after spike emission for the GIF model (defined in METHODS; $\mathrm{mV}$ )

\section{Linearization of conductance-based models}

The starting point for the analysis in this paper is the conductancebased Hodgkin-Huxley formalism. The state of a neuron is described by a potential difference $V$ across a membrane with a capacitance $C_{\mathrm{M}}$, a set of trans-membrane currents $I_{\text {mem }}$ (comprising the leak and various active ionic currents), a synaptic current $I_{\text {syn }}$ (to be described in the following text) and an applied current $I_{\text {app }}$

$$
C_{\mathrm{M}} \frac{\mathrm{d} V}{\mathrm{~d} t}=-I_{\mathrm{mem}}-I_{\mathrm{syn}}+I_{\mathrm{app}}
$$

The active ionic currents comprise both activation and inactivation variables $x_{k}$ where $k=1, \ldots, N$ counts over all the variables that obey equations of the form

$$
\tau_{k}(V) \frac{\mathrm{d} x_{k}}{\mathrm{~d} t}=x_{k, \infty}(V)-x_{k}
$$

where both the relaxation times $\tau_{k}(V)$ and the steady-state values $x_{k, \infty}(V)$ are functions of the membrane voltage.

Below threshold for action potential generation, Eqs. 1 and 2 can be linearized around a holding voltage $V^{*}$ (see e.g., Koch 1999, chapter 10 , and refs therein). For the sake of simplicity, the notation $X^{*}$ will be used to denote the quantity $X$ evaluated at $V=V^{*}$. Linearization of the Eq. set 1 and 2 allows for a direct categorization of the range of behavior that a neuron exhibits in its response to small input currents, for example, the response to an oscillating or square-pulse current considered here. The linearized equations will also provide the basis for a generalization of the IF model, to be described at the end of this section. The linear equations can be written in the following form

$$
\begin{gathered}
C_{\mathrm{M}} \frac{\mathrm{d} v}{\mathrm{~d} t}=-g_{\mathrm{M}} v-\sum_{k=1}^{N} g_{K} w_{K}-I_{\mathrm{syn}}+I_{\text {app }} \\
\tau_{k} \frac{\mathrm{d} w_{k}}{\mathrm{~d} t}=v-w_{k} \quad \text { where } \quad k=1, \ldots, N
\end{gathered}
$$

with $v=V-V^{*}$ being the deviation of the voltage from its steady-state value and $g_{\mathrm{M}}=\left(\partial I_{\mathrm{mem}} / \partial V\right)^{*}$ is the slope of the instantaneous $I$ - $V$ curve. The time-dependent variables

$$
w_{k}=\left(x_{k}-x_{k}^{*}\right) /\left(\frac{\mathrm{d} x_{k, \infty}}{\mathrm{d} V}\right)^{*} \text { for } k=1, \ldots, N
$$

are proportional to the deviation of the activation or inactivation variables $x_{k}$ from their steady-state values $x_{k}^{*}=x_{k, \infty}\left(V^{*}\right)$ and are expressed in units of millivolts. The time constants $\tau_{k}$ correspond to those of the activation and inactivation variables evaluated at $V^{*}$ and the parameters

$$
g_{k}=\left(\frac{\partial I_{\mathrm{mem}}}{\partial x_{k}}\right)^{*}\left(\frac{\mathrm{d} x_{k, \infty}}{\mathrm{d} V}\right)^{*}
$$

written in units of conductance, measure the strength of the effect that the variable $x_{k}$ has on the voltage. Note that in the linear approximation, the dynamical variables $w_{k}$ are no longer multiplied by a voltagedependent term as they were in the original conductance-based description.

Activation or inactivation variables can be classified according to the sign of their corresponding parameter $g_{k}$. Examples of variables with $g_{k}<0$ are the activation variables of $\mathrm{Na}^{+}$and $\mathrm{Ca}^{2+}$ currents and inactivation variables of $\mathrm{K}^{+}$currents. Examples of variables with $g_{k}>0$ include inactivation variables of $\mathrm{Na}^{+}, \mathrm{Ca}^{2+}$ currents, activation variables of $\mathrm{K}^{+}$currents, and the activation variable of the $\mathrm{H}$ current. For $g_{k}>0$, the corresponding variable opposes voltage change (negative feedback), whereas $g_{k}<0$ indicates that the variable 
amplifies voltage change (positive feedback). Previous modeling studies (reviewed in Hutcheon and Yarom 2000) have shown that a variable with $g_{k}>0$ can create a subthreshold resonance (a resonant variable), whereas a variable with $g_{k}<0$ can amplify an existing resonance (an amplifying variable).

Conductance-based models generally comprise many active ionic currents and are therefore described by a large number of activation or inactivation variables. Despite the simplification of linearity, such systems of equations can still be hard to handle analytically. However, it is often possible to reduce the number of variables to two or three, while still accurately modeling the behavior near the holding voltage. This can be achieved by considering that very fast variables (such as the activation variable of fast sodium channels) are instantaneous, by merging together variables with similar time constants, and by noting that very slow variables average over the voltage to provide a steady current. The resulting equations have the same form as $E q .3$ but with effective values $C$ and $g$ for the capacitance and leak respectively. The effective leak $g$ can be zero or even negative, while the resting potential remains stable. Examples of the linearization method are given in the APPENDIX for two conductance-based models together with the further approximations leading to reductions in the number of variables to two or three.

TWO-VARIABLE SUBTHRESHOLD DYNAMICS. For the case of two variables, the neuron is described by the two equations

$$
\begin{gathered}
C \frac{\mathrm{d} v}{\mathrm{~d} t}=-g v-g_{1} w+I_{\mathrm{app}}(t) \\
\tau_{1} \frac{\mathrm{d} w}{\mathrm{~d} t}=v-w
\end{gathered}
$$

with four parameters, $C, g, g_{1}$, and $\tau_{1}$. However, expressing time in units of $\tau_{1}$, and dividing the voltage $E q .6$ by $C$ makes it apparent that the model only depends on two dimensionless parameters $\alpha=g \tau_{1} / C$ and $\beta=g_{1} \tau_{1} / C$. The quantities $\alpha$ and $\beta$ parameterize the behavior of the neuron near $V^{*}$ and can be considered as representing a point on a plane. $\alpha$ represents an effective leak, whereas $\beta$ represents an effective coupling between the two variables. $\beta$ measures the influence of the $w$ variable on the membrane potential.

THREE-VARIABLE SUBTHRESHOLD DYNAMICS. The analysis is also extended to include a third variable. The subthreshold dynamics is then described by

$$
\begin{gathered}
\mathrm{C} \frac{\mathrm{d} v}{\mathrm{~d} t}=-g v-g_{1} w_{1}-g_{2} w_{2}+I_{\text {app }}(t) \\
\tau_{1} \frac{\mathrm{d} w_{1}}{\mathrm{~d} t}=v-w_{1} \\
\tau_{2} \frac{\mathrm{d} w_{2}}{\mathrm{~d} t}=v-w_{2}
\end{gathered}
$$

where in this paper a restriction is made to $g>0$. Four independent and dimensionless parameters $g_{1} / g, g_{2} / g, \tau_{1} g / C$, and $\tau_{2} g / C$ are now needed to fully describe the model.

\section{Models of spiking neurons}

One of the major goals of the present paper is to investigate the effect of subthreshold resonance on the dynamics of spike emission. To this end, a simple spiking neuron model that exhibits subthreshold resonance, the generalized IF neuron, is introduced. To demonstrate that the general results derived for this simplified model carry over to more realistic neurons, two representative conductance-based models that are known from the literature to produce subthreshold resonance are also examined (Models $I$ and $I I$ ). In an attempt to cover the range of possible behaviors, the models are chosen to have different resonance mechanisms (hyperpolarization or depolarization activated currents) and also different resonant frequencies (near 10 and $50 \mathrm{~Hz}$, respectively).

GENERALIZED IF NEURON. The IF model neuron provides a powerful tool for the understanding of neurons with passive membrane properties and is the standard component of large numerical simulations of recurrent networks. However, its passive subthreshold behavior cannot capture the phenomenon of resonance. An extension of the IF model, which captures the subthreshold behavior of the twovariable model with a simple spike mechanism, is therefore the first spiking neuron model to be introduced here. The generalized IF (GIF) neuron is obtained by supplementing $E q .3$ with a threshold for spike generation at $v=v_{\theta}$, followed by a reset of the membrane voltage at $v=v_{r}$ (the auxiliary variables $w_{k}$ considered here have a slower dynamics than the spike, and therefore it is not appropriate to reset them also). In the case $g_{k}=0$ for all $k$, the voltage equation reduces to the IF model. With two variables, this model is similar to a model recently proposed by Izhikevich (2001). The two-variable GIF model subject to an applied current is described by Eq. 6 where the parameters $C, g, g_{1}$, and $\tau_{1}$ are kept fixed for the whole of the subthreshold regime. $I_{\text {syn }}$ is the modeled synaptic current and $I_{\text {app }}(t)$ represents the applied current, to be described in the following text. In this paper, the threshold is chosen to be at $-50 \mathrm{mV}$, the rest (in absence of any input currents) at $-70 \mathrm{mV}$, and the reset at $-56 \mathrm{mV}$. Because $v$ measures the deviation from rest $v=V-V_{\text {rest }}$, this corresponds to $v_{\theta}=20 \mathrm{mV}$ and $v_{r}=14 \mathrm{mV}$.

\section{Conductance-based neurons}

MODEL I. A NEURON WITH $I_{\mathrm{NA}}, I_{\mathrm{K}}$, AND $I_{\mathrm{H}}$ CURRENTS. The first model comprises a hyperpolarization-activated mixed cation current $I_{\mathrm{H}}$ and the Hodgkin-Huxley spike-generating currents. The form of the $I_{\mathrm{H}}$ current is taken from Spain et al. (1987) and comprises both fast $f$ and slow $s$ activation variables. The time scales of the two components are $\tau_{f}=38 \mathrm{~ms}$ and $\tau_{s}=319 \mathrm{~ms}$ and, as in Spain et al. (1987), taken to be voltage independent. The fast component has the greater contribution and determines the resonant frequency $f_{\mathrm{R}}$, which is near $10 \mathrm{~Hz}$ at physiological temperatures. A detailed model description can be found in the APPENDIX.

MODEL II. A NEURON WITH $I_{\mathrm{NA}}, I_{\mathrm{K}}, I_{\mathrm{NAP}}$, AND $I_{\mathrm{KS}}$ CURRENTS. In contrast to the $I_{\mathrm{H}}$ model defined in the preceding section, the second model neuron features two depolarization-activated currents: the slow potassium current $I_{\mathrm{Ks}}$ and the persistent sodium current $I_{\mathrm{NaP}}$. In the language of Hutcheon and Yarom (2000), the $I_{\mathrm{Ks}}$ current generates the resonance and the $I_{\mathrm{NaP}}$ current amplifies its effect. A noninactivating form (Gutfreund et al. 1995) is used for the $I_{\mathrm{Ks}}$ with an activation time scale of $\tau_{q}=6 \mathrm{~ms}$ (Wang 1993), giving a subthreshold resonance that is strongest at resonant frequencies $\sim 35-55 \mathrm{~Hz}$. Neurons with a resonance frequency or subthreshold oscillations at $\sim 40 \mathrm{~Hz}$ are widespread (Pike et al. 2000; Puil et al. 1986), and the underlying mechanism is thought to sometimes involve the $I_{\mathrm{Ks}}$ and $I_{\mathrm{NaP}}$ currents (Llinas et al. 1991). Again, full details of this model are given in the APPENDIX.

\section{Modeling the noisy synaptic input}

The massive synaptic bombardment received by neurons in vivo represents a strong source of noise. Destexhe et al. (2001) provided evidence that an appropriate model of such a synaptic input is given by a fluctuating conductance with short correlation times related to the shapes of typical excitatory and inhibitory postsynaptic potentials. Hence, for the analysis of the firing rates of the two conductancebased models I and II, the noise is modeled as in Destexhe et al. (2001) by the equations 


$$
\begin{gathered}
I_{\text {syn }}=g_{\mathrm{e}}(t)\left(V-E_{\mathrm{e}}\right)+g_{\mathrm{i}}(t)\left(V-E_{\mathrm{i}}\right) \\
\tau_{\mathrm{e}} \frac{\mathrm{d} g_{\mathrm{e}}}{\mathrm{d} t}=g_{\mathrm{eo}}-g_{\mathrm{e}}+\sigma_{\mathrm{e}} \sqrt{2 \tau_{\mathrm{e}}} \xi_{\mathrm{e}}(t) \\
\tau_{\mathrm{i}} \frac{\mathrm{d} g_{\mathrm{i}}}{\mathrm{d} t}=g_{\mathrm{io}}-g_{\mathrm{i}}+\sigma_{\mathrm{i}} \sqrt{2 \tau_{\mathrm{i}}} \xi_{\mathrm{i}}(t)
\end{gathered}
$$

where $\xi_{\mathrm{e}}, \xi_{\mathrm{i}}$ are delta-correlated Gaussian white-noise terms. Representative values of the correlation times $\tau_{\mathrm{e}}=3 \mathrm{~ms}$ and $\tau_{\mathrm{i}}=10 \mathrm{~ms}$ are used here. The reversal potentials are taken to be $E_{\mathrm{e}}=0 \mathrm{mV}$ and $E_{\mathrm{i}}=$ $-75 \mathrm{mV}$. The average conductances $g_{\text {eo }}, g_{\text {io }}$ and the noise amplitudes $\sigma_{\mathrm{e}}$ and $\sigma_{\mathrm{i}}$ can be varied to explore a range of input conditions.

MODELING NOISE FOR THE GIF NEURON. To have a simple model with a membrane-potential-independent subthreshold resonance, the synaptic inputs are modeled by a current comprising a direct drive $I_{0}$ and a white-noise source

$$
I_{\text {syn }}=I_{0}+I_{\mathrm{N}} \sqrt{\tau_{\mathrm{N}}} \xi(t)
$$

where $\xi(t)$ is the delta-correlated Gaussian white-noise term with unit variance and $I_{\mathrm{N}}$ is the measure of the noise strength in nanoAmpere. The factor $\tau_{\mathrm{N}}$ is introduced to preserve units, and throughout this paper, it is arbitrarily fixed at $\tau_{\mathrm{N}}=1 \mathrm{~ms}$ without affecting the generality of the results. The noise strength $I_{\mathrm{N}}$ can be related to a more intuitive measure: the SD of the membrane voltage (in the absence of the spiking mechanism). In the two-variable GIF model, the SD of the voltage $\sigma_{\mathrm{V}}$ takes the form

$$
\sigma_{\mathrm{V}}=I_{\mathrm{N}} \sqrt{\frac{\left(C+g \tau_{1}+g_{1} \tau_{1}\right) \tau_{\mathrm{N}}}{2 C\left(g+g_{1}\right)\left(g \tau_{1}+C\right)}}
$$

\section{Response of the neuron to an oscillatory drive}

SUBTHRESHOLD RESPONSE. To characterize the subthreshold response, an oscillating current of frequency $f$ is used. The applied current and resulting voltage response to this current are given by

$$
\begin{gathered}
I_{a p p}=I_{0}+I_{1} \sin (2 \pi f t) \\
V=V^{*}+V_{1}(f) \sin (2 \pi f t+\theta(f))
\end{gathered}
$$

where both the phase difference $\theta(f)$ and the magnitude of the impedance

$$
|\mathrm{Z}(f)|=V_{1}(f) / I_{1}
$$

are functions of the driving frequency $f$. The existence of a peak in the $|Z(f)|$ versus frequency curve provides the definition of subthreshold resonance. The impedance $Z(f)$ can also be measured experimentally using a ZAP current (Puil et al. 1986).

FIRING-RATE RESPONSE. In the context of examining the interactions between membrane frequency preference (resonance) and network oscillations, it is of interest to examine how the instantaneous firing rate of a neuron responds to a sine-wave modulation in the background of a noisy synaptic current

$$
I_{\text {app }}=I_{1} \sin (2 \pi f t)
$$

A regime is considered where the noisy synaptic drive is sufficiently strong to cause the neuron to fire stochastically, at an average rate $r_{0}$. The weak sinusoidal component then causes a weak modulation of the firing rate that will be apparent over many trials, see Fig. 1. This quantity can also be thought of as the firing rate, averaged over a population of neurons each individually receiving a noisy drive but responding collectively to the same weak oscillatory component present in the firing rates of presynaptic neurons. The form of this population, or trial-averaged instantaneous rate is

$$
r=r_{0}+r_{1}(f) \sin (2 \pi f t+\phi(f))
$$
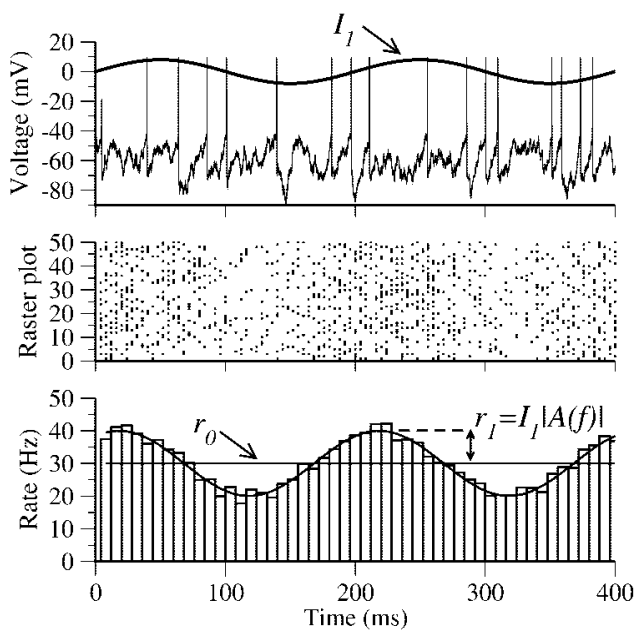

FIG. 1. The response of the instantaneous firing rate to a weak sinusoidal input. The oscillatory current is applied on top of a noisy input that itself elicits firing at an average rate $r_{0}$ (see top). By averaging over many realizations (shown in the raster plot), the modulation of the instantaneous firing rate can be computed, and the characteristics (amplitude and phase) of the induced sinusoidal component of the firing rate obtained (bottom). A bin width of $8 \mathrm{~ms}$ was used for illustrative purposes in this figure.

The analogy with the subthreshold voltage form in Eq. 11 is clear. Similarly, the response $r_{1}(f)$ is proportional to the strength of the modulatory current, leading to the introduction of the following quantity that measures the ability of a neuron to amplify a particular frequency

$$
|A(f)|=r_{1}(f) / I_{1}
$$

called the signal gain, see for example (Gerstner 2000). In the same way that a peak in the impedance $|Z(f)|$ quantifies subthreshold resonance, the existence and position of the peak in the quantity $|A(f)|$ will be the corresponding firing-rate measure of resonance.

Experimental studies have used either large amplitude sine-wave currents (Hutcheon et al. 1996b) or small-amplitude sine-wave currents when the voltage is very close to threshold (Pike et al. 2000). In both of these studies, a strong effect was measured in the time-averaged rate itself and not in its modulation. The situation considered here is of weak oscillatory input leading to a linear response of the firing rate (higher-order harmonics are negligibly small). In this case, the signal gain is a more sensitive measure of the frequency dependence of spike emission than the time-averaged firing rate.

ANALYTICAL METHODS. The firing-rate response of the GIF neuron can be computed analytically in the limit of large $\tau_{1}$. Methods are sketched in the APPENDIX (see also Brunel et al. 2003).

NUMERICAL METHODS. The numerical analysis of the firing-rate response of the GIF and conductance-based models was performed using a stochastic second-order Runge-Kutta algorithm (Honeycutt 1992) with a time step of 10 and $20 \mu$ s, respectively. The amplitude of the modulatory current $I_{1}$ used in numerical measurements of the signal gain was varied until it was sufficiently small such that higher order nonlinear effects were negligible. The length of simulation time needed to get accurate measurements for each frequency point varied between 1,000 and $50,000 \mathrm{~s}$, depending on the firing rate and the particular level of noise chosen in the input current. To estimate the firing rate modulation at a given frequency, the instantaneous firing rate is computed in bins of $1 \mathrm{~ms}$. The resulting histogram, sketched in Fig. 1, is then fitted by a sinusoid with a frequency equal to that of the oscillatory input current. 
RES ULT S

\section{Subthreshold properties of the membrane potential}

The different classes of behavior in the subthreshold regime are examined first. Two- and three-variable models, with parameters directly related to measurable membrane properties, are used to classify the different types of response to standard test currents. It is shown that the types of behavior that the neuron can exhibit at different holding voltages can be conveniently presented in graphical form for both the two- and three-variable descriptions. The results are illustrated by two conductance-based models of spiking resonant neurons.

\section{Subthreshold behavior of the two-variable model}

The subthreshold behavior of the two-variable model is first classified with respect to stability and the response to standard test currents. Because the two-variable model is related to an underlying conductance-based description near a holding voltage $V^{*}$, it is parameterized by an effective leak $\alpha=g \tau_{1} / C$ and an effective coupling between the two variables $\beta=g_{1} \tau_{1} / C$. The parameters $\alpha$ and $\beta$ can be used to represent the behavior of the neuron by a string of points on a plane, as the neuron is depolarized or hyperpolarized by an injected current. The borders separating different types of behavior are obtained through the analysis of Eq. 6, presented in detail in the APPENDIX.

STABILITY. The classification of the subthreshold regime starts with the determination of the parameter region where the neuron remains stable at the holding potential (without e.g., subthreshold oscillations or spike emission). Analysis of the stability of the membrane potential of the two-variable model determines an unstable region shown in brown in Fig. 2. The region is bounded on one side by the vertical dashed line that signals the onset of spontaneous oscillations. On the other side, it is bounded by a diagonal line that corresponds to the total input conductance becoming zero, which can lead to spike emission. The rest of the analysis will focus on the stable region to the right of these two lines.

RESPONSE TO OSCILLATING CURRENT. The first experimental measure of subthreshold properties considered here is the voltage response to an oscillating input current. The magnitude and phase of this response measure the impedance of the neuronal membrane. A subthreshold resonance, signaled by the existence of peak in $|Z(f)|$ at some nonzero frequency $f_{\mathrm{R}}$, occurs in the whole of the green region of Fig. $2 A$ (Hutcheon et al. 1996a). The line that bounds the region of the phase diagram in which resonance occurs starts at the point $\alpha=-1, \beta=1$ (the intersection between the two instability lines), and for large $\alpha$, it tends toward the axis $\beta=0$. Thus in most of the stable region with $\beta>0$ resonant behavior occurs. Positivity of $\beta$ implies that the associated activation or inactivation variable is a resonant variable (Hutcheon and Yarom 2000).

PHASE RESPONSE TO OSCILLATING CURRENT. Another quantity of interest is the existence of a zero phase-lag in the membrane potential response at nonzero frequency, seen in cortical neurons (Gutfreund et al. 1995). Analysis of the phase difference $\theta(f)$ between the oscillating current and the voltage response, defined in Eq. 11, shows that a zero phase-lag exists for $\beta>$ 1. This quantity also coincides with the existence of a maxi-
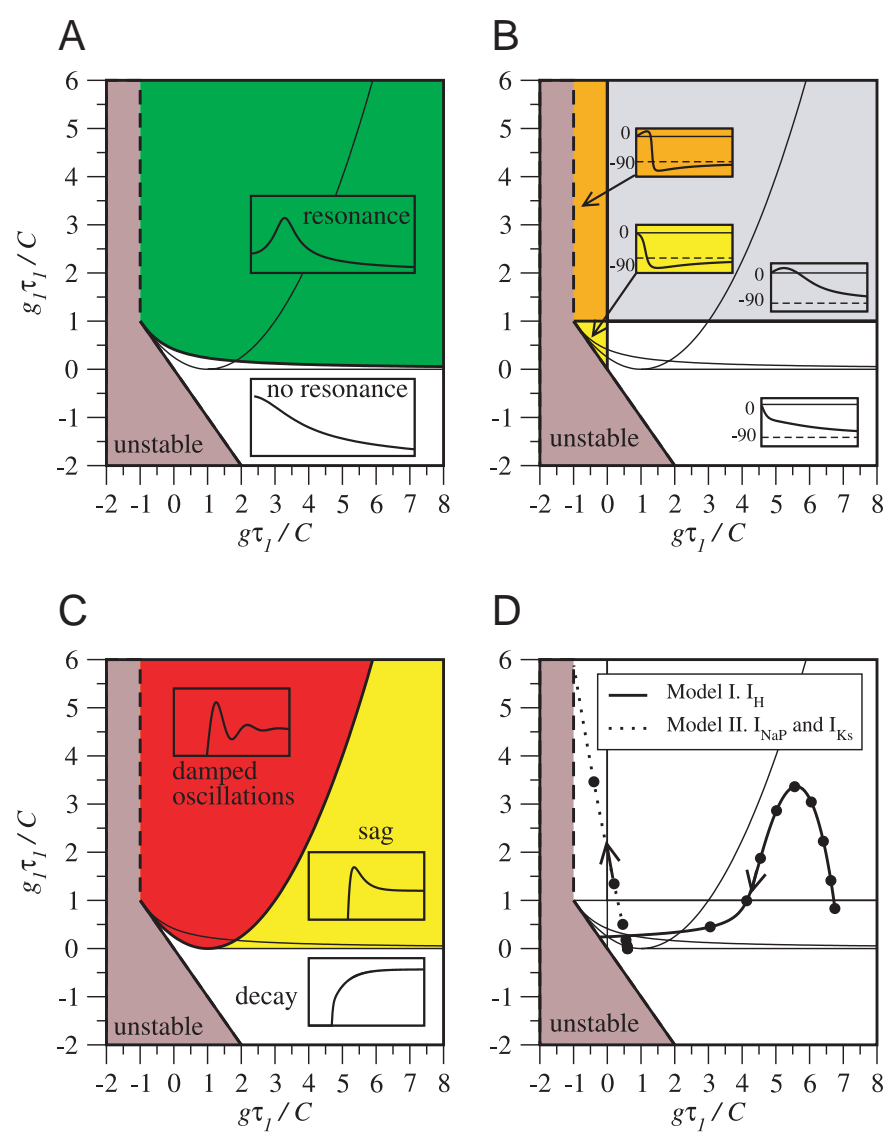

FIG. 2. The subthreshold behavior of two-variable models. Brown marks the unstable region. Insets: the qualitative response to the relevant test current in regions in the space of parameters $\alpha=g \tau_{1} / C$ (the effective leak) and $\beta=$ $g_{1} \tau_{1} / C$ (the effective coupling between the two variables). $A$ : oscillating current injection, amplitude of the impedance. $B$ : phase of the impedance. $C$ : response to a square-pulse current. $D$ : trajectories of the two conductancebased model neurons in parameter space as their holding potential is increased. Model I (full line): $g_{1}=g_{f}$ (see text and APPENDIX) parameterizes the effect of the fast component of the current $I_{\mathrm{H}}$ on the voltage. The trajectory covers the range from -100 to $-56.5 \mathrm{mV}$ at which point the resting state is unstable and spikes are emitted. Model II (dotted line): $g_{1}=g_{q}$ (see text and APPENDIX) parameterizes the effect of the slow-potassium current $I_{\mathrm{Ks}}$ on the voltage. The trajectory covers the range -100 to $-57 \mathrm{mV}$ at which point spontaneous oscillations occur. In both cases, the black points are at 5-mV intervals with the last point plotted before destabilization occurring at $-60 \mathrm{mV}$, and the arrows represent the direction of depolarizing membrane potential.

mum in the phase and a phase advance from the driving current. A second line $\alpha<0$ signifies the existence of a minimum in the phase, implying that at some frequencies the phase lag is greater than $90^{\circ}$. Taken together, these criteria divide the phase diagram into four regions, plotted in Fig. $2 B$. It should be noted that none of the lines separating the different qualitative responses of the phase correspond exactly to the presence of a resonance in the amplitude of the impedance.

RESPONSE TO A SQUARE-PULSE CURRENT. The application of a small step change in applied current provides a different assessment of subthreshold membrane properties. The response of the neuron to such a current can be obtained explicitly (see APPENDIX for details). At the level of the two-variable description the neuron can exhibit three different types of response to the square-pulse current shown, the voltage-time profiles of which are shown in the insets of Fig. $2 C$ : 
Damped oscillations. The neuron exhibits damped oscillations at frequency $f_{0}$ as it approaches its new holding membrane potential, when $\beta$ is sufficiently large compared with $\alpha$ (the red region shown in Fig. 2C).

Overshoot or sag. When $\alpha>1$ and $\beta>0$, but below the red region, the voltage time course has a single overshoot (or sag if the current pulse is hyperpolarizing). This corresponds to the yellow region of Fig. $2 C$.

Passive decay. In all other areas of parameter space (the white region), the voltage changes monotonically from its initial to final resting voltage.

It is clear from Fig. 2, $A$ and $C$, that subthreshold resonance and damped oscillations are not equivalent. This fact, which was implicit in experimental measures of the resonant and natural frequencies (Puil et al. 1986), is often overlooked. An examination of Fig. 2 shows that neurons can have damped oscillations but no resonance and vice versa. In fact none of the other measurements (the phase or response to a square-pulse current) of the neuron examined here give complete information about the existence of a resonance. However, close to the instability line, where the neuron is almost spontaneously oscillating, both resonance and damped oscillations are guaranteed to occur together.

\section{Subthreshold behavior of the two conductance-based models}

The diagram introduced in Fig. 2 allows a visualization of the trajectory of the neuron through the space of parameters $\alpha\left(V^{*}\right)$ and $\beta\left(V^{*}\right)$ as the holding voltage $V^{*}$ is varied. This is shown for the two model neurons defined in METHODS and detailed in the APPENDIX. As the trajectory crosses different boundaries, so the neuronal response to input current will change qualitatively. The trajectories of the two model neurons, as parameterized by the changing effective leak $g$ and coupling variable $g_{1}=g_{f}$ or $g_{1}=g_{q}$, are plotted in Fig. $2 D$.

MODEL I. A NEURON WITH $I_{\mathrm{NA}}, I_{\mathrm{K}}$. AND $I_{\mathrm{H}}$ CURRENTS. The resonance curve of the neuron is plotted in Fig. $3 A$ for a

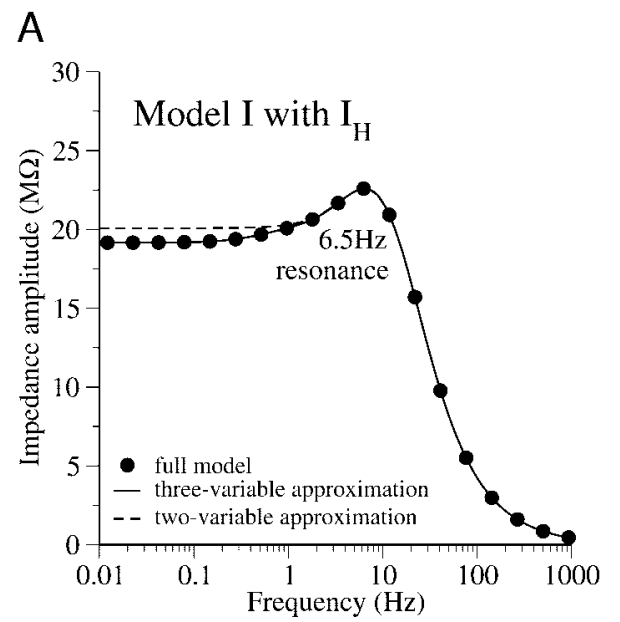

holding potential of $-65 \mathrm{mV}$. The spike-generating currents are much faster than other time scales in the system and can be taken as instantaneous, reducing the full model to a threevariable description: the membrane potential and the two activation variables of the $\mathrm{H}$ current (see APPENDIX for details). The impedance curve of the reduced three-variable description is also shown in Fig. 3A. Comparison with the full model shows that this reduction is extremely accurate. A further approximation, that the slow variable of the $I_{\mathrm{H}}$ current averages to a steady value, provides the two-variable description. The behavior of the neuron is therefore classified by its leak conductance $g$ and the effect of the $I_{\mathrm{H}}$ fast variable $f$, through the two dimensionless parameters $\alpha=g \tau_{f} / C$ and $\beta=g_{f} \tau_{f} / C$. This two-variable description provides an excellent approximation of the original model for driving frequencies greater than $2 \mathrm{~Hz}$ as shown in Fig. 3A. At frequencies greater than $2 \mathrm{~Hz}$, the dynamics of the slow variable of the $\mathrm{H}$ current is too slow to follow the voltage changes and therefore to affect the resonance curve.

The effective leak $\alpha$ and effective coupling between voltage and $\mathrm{H}$ current $\beta$ are calculated for a subthreshold voltage range of -100 to $-56.5 \mathrm{mV}$ using the linearization procedure. The corresponding trajectory is shown in Fig. $2 D$, and the resonance and damped oscillation frequencies are shown in Fig. $4 A$. As can be seen, model I exhibits a strong resonance at hyperpolarized values in the absence of damped oscillations (except in a narrow range between -58.5 and $-56.6 \mathrm{mV}$ near the firing threshold). This illustrates again that resonance and damped oscillations are distinct phenomena: oscillating and step currents probe different membrane properties. The $Q$ value, defined as $\left|Z\left(f_{R}\right)\right| /|Z(0)|$ (Hutcheon et al. 1996b), gives a measure of the strength of the resonance. As can be seen in Fig. $4 A$, the $I_{\mathrm{H}}$ current provides the strongest resonance of $\sim 10 \mathrm{~Hz}$ at a holding voltage of $-80 \mathrm{mV}$. The trajectory also shows that the neuron responds with a sag/rebound to a step-current pulse: a well-known characteristic of the $I_{\mathrm{H}}$ current (Dickson et al.

B

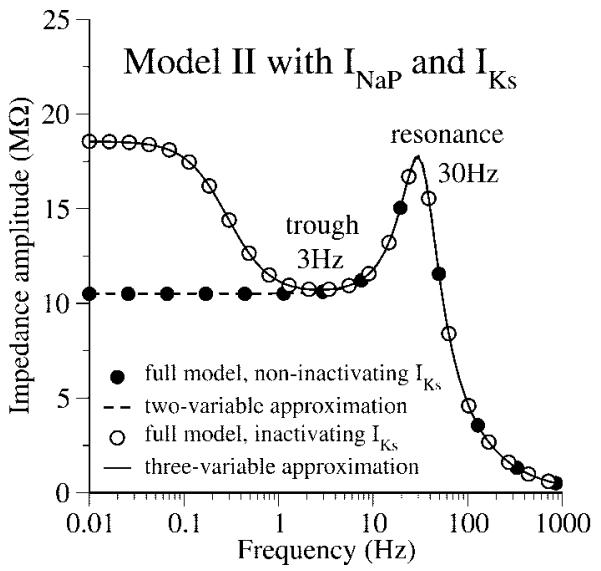

FIG. 3. The frequency-dependent input impedance for the two model neurons held at $-65 \mathrm{mV}$, showing the level of approximation between the full ( $\bullet$ and $\mathrm{O})$, three-variable $(-)$ and two-variable (- - ) descriptions. A: model I. The three-variable model is obtained by taking the spike-generating currents to be instantaneous. The full and three-variable models agree closely for all frequencies plotted. The two-variable approximation is obtained by noting that the slow variable of the $I_{\mathrm{H}}$ current averages to a steady value for frequencies greater than $\sim 2 \mathrm{~Hz}$. B: model II. The full noninactivating $I_{\mathrm{Ks}}$ model $(\bullet)$ and its two-variable approximation, obtained by taking the spike-generating currents to be fast. If an inactivation variable is included in the definition of the $I_{\mathrm{Ks}}$ current, an impedance profile (O) with a trough at $3 \mathrm{~Hz}$ as well as a resonance at $30 \mathrm{~Hz}$ is seen (see Three-variable model and the APPENDIX for details). The corresponding three-variable model obtained by taking the spike-generating currents to be fast, but retaining the activation and inactivation variables of the $I_{\mathrm{Ks}}$ current, provides a good approximation of the full model. 
A

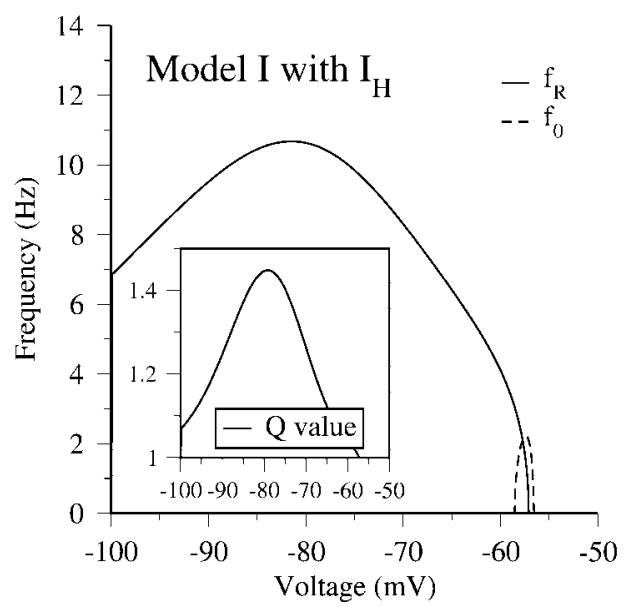

$\mathrm{B}$

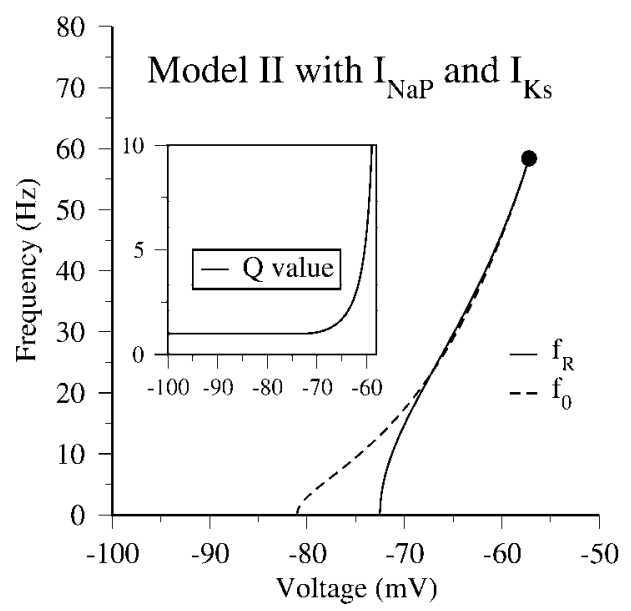

FIG. 4. The resonance frequency $f_{R}(-)$ and frequency of damped-oscillations $f_{0}(---)$ of the 2 model neurons as a function of holding voltage. The corresponding $Q$ values are also given that, following convention, measure the relative strength of the resonant peak $Q=$ $\left|Z\left(f_{R}\right)\right| /|Z(0)|$. A: model I. A resonance exists for most of the subthreshold regime, whereas damped oscillations occur only in a narrow voltage range near the firing threshold (at $-56.4 \mathrm{mV}$ ). The resonance is strongest at -80 $\mathrm{mV}$ with $f_{\mathrm{R}}=10 \mathrm{~Hz} . B$ : model II. In this case, both resonance and damped oscillations exist above $-72.5 \mathrm{mV}$. The resonance strength increases as the membrane potential approaches the onset of spontaneous oscillations (indicated by $\bullet$ at $-57.2 \mathrm{mV}$ ), above which the neuron fires periodically. The regions in which damped oscillations and resonance exist shown in these graphs can be compared with the trajectories in Fig. $2 D$.
2000). As the holding voltage is increased to more depolarized values the $I_{\mathrm{H}}$ current weakens until the resonance vanishes at around $-57 \mathrm{mV}$.

MODEL II. A NEURON WITH $I_{\mathrm{NA}}, I_{\mathrm{K}}, I_{\mathrm{KS}}$, AND $I_{\mathrm{NAP}}$ CURRENTS. In a similar way to the previous case, the full conductance-based model can be reduced to a two-variable description by noting that the spike-generating currents are fast. This approximation is very accurate, as can be seen in Fig. $3 B$ (the noninactivating $I_{\mathrm{Ks}}$ and two-variable profiles). The behavior of this neuron is classified by its leak $g$, the $I_{\mathrm{Ks}}$ coupling variable $g_{\mathrm{q}}$, and its time constant $\tau_{q}$ through the dimensionless parameters $\alpha=g \tau_{\mathrm{q}} / C$ and $\beta=g_{q} \tau_{q} / C$. These quantities are calculated for a subthreshold voltage range of -100 to $-57 \mathrm{mV}$, and the corresponding trajectory is plotted in Fig. $2 D$. In distinction to model I, the resonance here is driven by depolarization-activated currents. This can be seen in the vertical-moving trajectory and the increasing $Q$ value as the line of onset of spontaneous oscillations is approached. This neuron features both a resonant current in the activation of $I_{\mathrm{Ks}}$ as well as an amplifying current $I_{\mathrm{NaP}}$. The amplification effect is clearly seen in a comparison of the $Q$ values of the resonance of model II with model I (which lacks an amplifying mechanism). An examination of Fig. $4 B$ shows that the resonant frequency steadily increases as the line of onset of spontaneous oscillations is approached, a feature reminiscent of the "broad-frequency cells" in Llinas et al. (1991) that were also shown to feature a TTX-sensitive persistent sodium current as well as a delayed rectifier. The amplification is due to the fact that the $I_{\mathrm{NaP}}$ current decreases the effective leak (because the current amplifies voltage changes). Hence, the parameter $\alpha$ decreases as an effect of the activation of this current, and correspondingly the model moves toward the left in the diagram of Fig. $2 D$, in the direction of the line where spontaneous oscillations occur. On this line, the $Q$ value diverges.

\section{Subthreshold behavior of the three-variable model}

Although two-variable models capture the properties of a broad class of neurons, Fig. $2 A$ shows that they only provide two classes of impedance curves, either monotonously decreasing with increasing frequency (nonresonant case shown in white) or with a single peak at a preferred frequency (resonance case shown in green). Therefore more complex imped- ance curves cannot be described with only two variables. For this reason, the same classification described in the preceding text was performed for a model with three variables defined in $E q$. 7. The model is now specified by four parameters: the conductance ratios $g_{1} / g$ and $g_{2} / g$ and the time constants $\tau_{1}$ and $\tau_{2}$ of the variables $w_{1}$ and $w_{2}$. Without loss of generality, the time constant of $w_{2}$ was chosen to be the faster variable, thus $\tau_{2}<\tau_{1}$. When the faster variable is taken to be instantaneous, $\tau_{2}=0$, the model becomes equivalent to the two-variable model with $\alpha=\left(g+g_{2}\right) \tau_{1} / C$ and $\beta=g_{1} \tau_{1} / C$.

The classification of the three-variable model was done in terms of the presence or absence of damped oscillatory behavior in response to transient inputs and presence or absence of resonant behavior as defined by the peaks of the impedance profile. Figure 5 shows the different regions of interest in the $\left(g_{1} / g\right)$ versus $\left(g_{2} / g\right)$ plane for several values of the times constants $\tau_{1}$ and $\tau_{2}$ relative to the time constant $\tau=\mathrm{C} / \mathrm{g}$.

STABILITY. As in the two-variable case, the equilibrium voltage may destabilize in two different ways: either the total conductance $g_{1}+g_{2}+g$ becomes negative (Fig. 5, - ), or spontaneous oscillations appear (Fig. 5, - - ). These two lines intersect at the point where the frequency of the spontaneous oscillations becomes zero. When the time constants are such that $\tau_{2} \ll \tau_{1}$, the line where spontaneous oscillations arise becomes vertical. Thus as stated in the preceding text, in the limit $\tau_{2}=0$, the behavior of the two-variable model is recovered (compare Fig. 5B with Fig. 2).

RESONANCE AND DAMPED OSCILLATIONS. Again, similar but distinct regions are observed in which damped oscillations and resonant behavior occur. In the upper-left quadrant of each panel (where the slower variable is "resonant," $g_{1}>0$, while the faster variable is "amplifying," $g_{2}<0$ ), both damped oscillations and resonant behavior are present in most of the stable region. In the upper-right quadrant where both variables are "resonant," damped oscillations and resonant behavior are found almost throughout. In the lower-left quadrant, both variables are amplifying: the neuron exhibits neither damped oscillations nor resonance and destabilizes exclusively by the total conductance becoming zero.

APPEARANCE OF A TROUGH. In the bottom-right quadrant (where the slower variable is now amplifying $g_{1}<0$ and the faster variable is resonant $g_{2}>0$ ), a qualitatively new phe- 
A
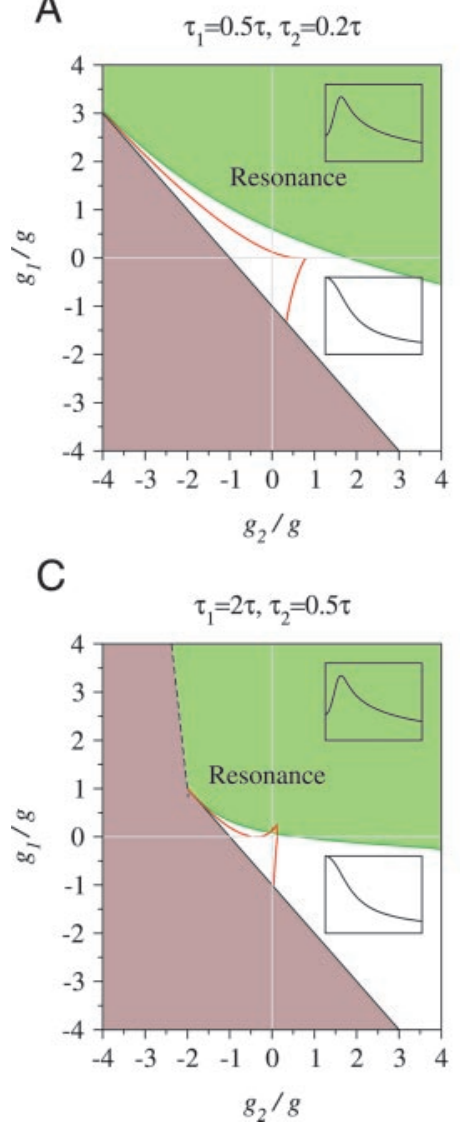

B $\quad \tau_{1}=5 \tau, \tau_{2}=0.2 \tau$

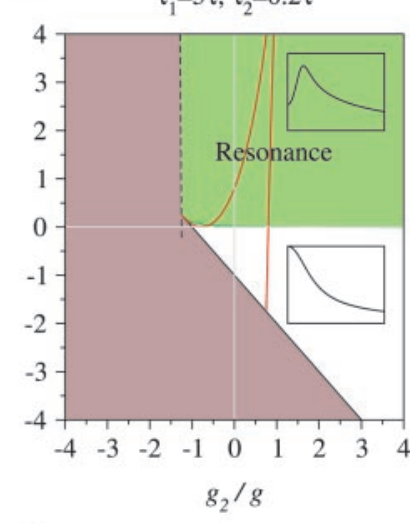

$\mathrm{D}$

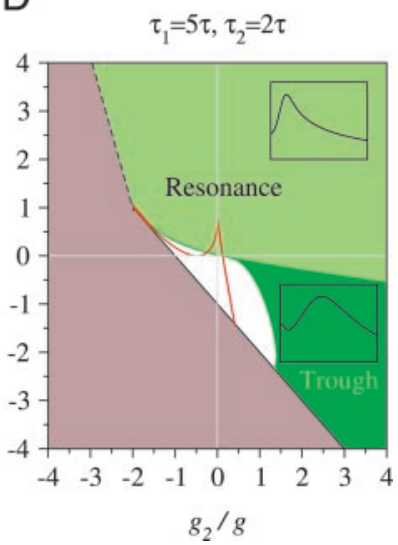

FIG. 5. Phase diagram of the three-variable model in the plane $g_{1} / g, g_{2} / g$, for different values of the time constants $\tau_{1}$ and $\tau_{2}$ relative to $\tau=C / g$ (marked above each panel). Brown, unstable region; green, regions where resonance occur; dark green, resonance with a trough at a lower frequency; red lines, the boundaries of the regions in which damped oscillations occur in response to a current step. See text for more details.

nomenon is observed, depending on the values of the time constants. When the two variables $\tau_{1}$ and $\tau_{2}$ are slower than the time constant $\tau=C / g$, the amplitude of the impedance has a local minimum or trough at a finite frequency (indicating a suppression of the membrane response at that frequency) followed by a resonant peak at higher frequency. One example of such subthreshold dynamics is a neuron with an inactivating potassium current with a relatively large activation time constant $\tau_{2}$ and a much larger inactivation time constant $\tau_{1}$ with overlapping steady-state activation and inactivation functions (a window current). In fact, a model of the $I_{\mathrm{Ks}}$ current that also includes an inactivation variable (see APPENDIX for details) gives exactly this effect. The frequency-impedance curve for such a neuron is plotted in Fig. $3 B$ and shows a close similarity to the experimentally measured impedance curve of the fastspiking interneurons measured in Pike et al. (2000). A two- and three-variable reduction of this full conductance-based model are also plotted for comparison. Another possibility would be to have the two variables implemented in two active persistent currents: a potassium current with activation time constant $\tau_{2}$ and a sodium or a calcium current with slower activation time constant $\tau_{1}$.

As expected, increasing the complexity of the model in terms of the number of descriptive variables also increases the range of neuronal behavior that can be modeled. In summary,

a one-variable model (like the subthreshold dynamics of the leaky IF neuron) can have only a monotonously decaying impedance; a two-variable model can have either a monotonously decaying impedance, or an impedance with a resonant peak, whereas a three-variable model can describe the two above-mentioned behaviors, and in addition, an impedance with a trough at low frequency followed by a peak at higher frequency.

\section{Firing-rate resonance}

In this section, the effect of the subthreshold resonance on the dynamics of the firing rate is investigated. The aim of the analysis is to determine when a small oscillatory component in the synaptic inputs of a given neuron will be amplified in its output and how this depends on the subthreshold properties of the considered neuron.

The current used to model the synaptic bombardment such a neuron would experience in vivo comprises a noisy hyperpolarizing or depolarizing drive as well as a weak sinusoidal component of frequency $f$. The signal gain $A(f)$ defined in $E q$. 14 and illustrated in Fig. 1 measures the strength of the temporal modulation of the instantaneous firing rate induced by the oscillating current. It is the firing-rate analog of the impedance $Z(f)$, and it is the existence of a peak in the signal gain that categorizes the amplification of frequencies in the outgoing spike train of resonant neurons. As will be shown, the noise inherent in biological networks is an important factor in determining the frequency that is maximally amplified.

The range of behavior is first examined by the use of a GIF model neuron. These results are then illustrated by two conductance-based models with spike generating currents and also an $I_{\mathrm{H}}$ current or $I_{\mathrm{NaP}}$ and $I_{\mathrm{Ks}}$ currents.

\section{Firing-rate resonance in the GIF model neuron}

In the previous section, the subthreshold behavior of a general two-variable model was analyzed in detail. As described in METHODS, a simple spike mechanism (threshold and reset) can be added to the two-variable description to produce a generalization of the IF neuron. This provides the simplest mathematical description of a spiking neuron with resonant subthreshold dynamics and allows a direct link to be made between the subthreshold characteristics and the statistical properties of the outgoing spike train. In spite of its simplicity, the GIF model provides a good approximation to more complete descriptions of neurons as will be seen in the next section.

The model examined here is parameterized by $C=0.5 \mathrm{nF}$, $g=0.025 \mu \mathrm{S}, g_{1}=0.025 \mu \mathrm{S}$, and $\tau_{1}=100 \mathrm{~ms}$, giving a subthreshold resonance frequency $f_{R}$ near $5 \mathrm{~Hz}$. The signal gain $A(f)$ was examined as a function of frequency for different respective strengths of the constant $I_{0}$ and noisy $I_{\mathrm{N}}$ components of the injected current, defined in Eq. 9. It should be noted that the sinusoidal component $I_{1}$ is always taken to be weak in the present work.

The fact that the neuron is induced to fire at a frequency $r_{0}$ by the applied current implies that there are now two distinct and independent frequency scales: the subthreshold resonant frequency $f_{\mathrm{R}}$, controlled by the subthreshold dynamics of the membrane potential, and the background firing frequency $r_{0}$, 

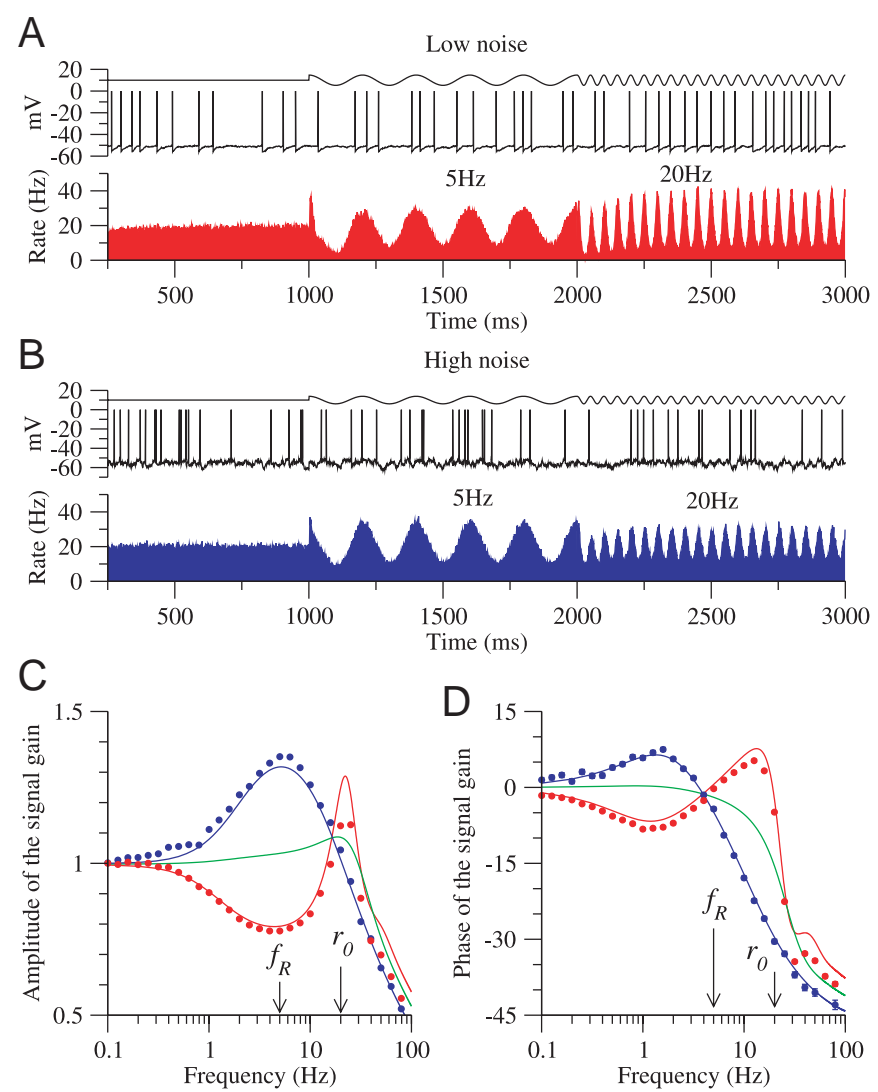

FIG. 6. Effect of noise strength on the amplification of a frequency $f$ in the firing rate of a resonant neuron. Two cases are considered: low noise (red) and high noise (blue). The particular generalized integrate-and-fire (GIF) model neuron $\left(C=0.5 \mathrm{nF}, g=0.025 \mu \mathrm{S}, g_{1}=0.025 \mu \mathrm{S}\right.$, and $\left.\tau_{1}=100 \mathrm{~ms}\right)$ has a resonance near $5 \mathrm{~Hz}$, and in all cases, the firing rate is kept at $r_{0}=20 \mathrm{~Hz}$. $A$ and $B$ : simulation of a 3 -s current injection protocol. $0-1 \mathrm{~s}$ : injected noisy current with no oscillatory component. 1-2 s: addition of a $5 \mathrm{~Hz}\left(f_{\mathrm{R}}\right)$ sinusoidal component. 2-3 s: sinusoidal component at $20 \mathrm{~Hz}\left(r_{0}\right)$. A: low-noise case $\left(I_{0}=\right.$ $0.95 \mathrm{nA}, I_{1}=0.024 \mathrm{nA}$, and $\left.I_{\mathrm{N}}=0.11 \mathrm{nA}\right)$ in which the neuron fires regularly. The amplification is greatest at $f=r_{0}=20 \mathrm{~Hz}$. $B$ : high-noise case $\left(I_{0}=0.78\right.$ $\mathrm{nA}, I_{1}=0.059 \mathrm{nA}$, and $\left.I_{\mathrm{N}}=0.55 \mathrm{nA}\right)$. The strongest amplification is at the resonance frequency $f=f_{\mathrm{R}}=5 \mathrm{~Hz}$. $C$ : signal gain amplitude $|A(f)|$ vs. frequency. $D$ : phase of the signal gain $\phi(f)$ vs. frequency. Both low (red curves) and high (blue curves) noise cases with the green curves representing an intermediate case $\left(I_{0}=0.92 \mathrm{nA}\right.$ and $I_{\mathrm{N}}=0.22 \mathrm{nA}$ giving $\left.\sigma_{\mathrm{V}}=1.36 \mathrm{mV}\right)$. The signal gain curves are normalized to 1 at $f=0$, see APPENDIX. The full lines are the theoretical predictions and the symbols come from numerical simulations of the model.

which is controlled by the characteristics of the externally applied noisy current. It is useful to distinguish situations depending on whether $r_{0}$ is lower or greater than $f_{\mathrm{R}}$.

High firing rate $r_{O}>f_{R}$. When the firing rate of the neuron is greater than its resonance frequency, two distinct modes of behavior were identified: when the neuron fires regularly due to a direct drive with a low-noise term (low noise) and when the neuron fires irregularly due to a high-noise term and a weak direct drive (high noise). Histograms of the response of these two cases to a current composed of a nonoscillating interval, an interval with a component of frequency $f_{\mathrm{R}}=5 \mathrm{~Hz}$ and a final interval with a component of frequency $r_{0}=20 \mathrm{~Hz}$ are plotted in Fig. 6, $A$ and $B$. The full frequency-dependent profiles of the signal gain $A(f)$ and phase $\phi(f)$ are also given in Fig. $6 C$ and $D$.
Low noise. The neuron fires regularly under the action of a strong direct drive $I_{0}=0.95 \mathrm{nA}$ perturbed by a weak noise term $I_{N}=0.11 \mathrm{nA}\left(\sigma_{\mathrm{v}}=0.68 \mathrm{mV}\right)$. In this regime (the red curves of Fig. 6), the neuron behaves as a nonlinear oscillator of frequency $r_{0}$. In the spike histogram (Fig. 6A), it is clear that the modulation is strongest in the third interval where the modulation of the current is at a frequency of $20 \mathrm{~Hz}$, equivalent to the firing rate.

High noise. In the converse case of noise-driven irregular firing (still at an average rate of $r_{0}=20 \mathrm{~Hz}$ ) with $I_{0}=0.78 \mathrm{nA}$ and $I_{\mathrm{N}}=0.55 \mathrm{nA}\left(\sigma_{\mathrm{V}}=3.4 \mathrm{mV}\right)$, the amplification properties of the resonant neuron change. As shown in the corresponding (blue) spike histogram of Fig. $6 B$, the amplification is now strongest in the second interval of the response, corresponding to a modulation of the input current at a frequency equal to the subthreshold resonance $f_{\mathrm{R}}=5 \mathrm{~Hz}$. Note that the noise level chosen here corresponds to the typical magnitude of membrane potential fluctuations measured in vivo, which are $\sim 3-5 \mathrm{mV}$ (Anderson et al. 2000; Destexhe and Paré 1999).

It is instructive to examine the signal gain for different values of noise, while the firing rate $r_{0}$ is kept constant through compensatory changes of the direct drive $I_{0}$, see Fig. $6 C$. As the relative strength of the noise is increased, the signal-gain peak at the firing rate decreases and vanishes before the peak at the resonance frequency emerges at higher noise levels. The green curve in Fig. $6 C$ shows an intermediate case with a flat signalgain profile. The curves showing the phase in Fig. $6 D$ show qualitatively similar behavior, except that the peaks (indicating the greatest phase advance) are at lower frequencies than the corresponding peaks in the signal gain.

Low firing rate $r_{O}<f_{R}$. When the firing rate $r_{0}$ is lower than the resonant frequency $f_{\mathrm{R}}$, the "low-noise" (regular firing) situation becomes almost impossible to reach because in absence of noise the neuron often starts to fire at a finite frequency (a type II neuron, see e.g., Rinzel and Ermentrout 1989). In this case, firing at low frequencies can only be achieved through the action of a noisy current, rendering neuronal firing irregular. Thus in practice, low firing rates imply high noise (noise-driven firing). When $r_{0}<f_{\mathrm{R}}$, the inter-spike interval is sufficiently long such that the neuron has time to explore its subthreshold regime and begin to "resonate" before firing. As could be expected, the subthreshold resonant frequency is therefore strongly evident in the signal gain, see Fig. $7 \mathrm{~A}$ in which the neuron was driven by a weak DC $I_{0}=$ $0.50 \mathrm{nA}$ and noise term $I_{\mathrm{N}}=0.55 \mathrm{nA}\left(\sigma_{\mathrm{V}}=3.4 \mathrm{mV}\right)$. The full profile of the signal gain demonstrates that the frequency most preferentially amplified is the subthreshold resonance $f_{\mathrm{R}}=5$ $\mathrm{Hz}$, as in Fig. $6 B$. This case is therefore similar to the highfiring-rate case (high noise) for which noise also dominated the firing process, despite the fact that the inter-spike dynamics are quite different.

The amplitude and phase of the signal gain $A(f)$ give a full picture of the changing behavior as the neuron moves between regular firing and irregular noise-driven firing in these two cases. As stated previously, the advantage of the GIF neuron is that it allows for a direct link between the subthreshold properties and their effect on the firing rate. A mathematical analysis, presented elsewhere (unpublished results), provides analytical expressions for the signal gain and its phase in terms of the parameters of the model, in the limit that $\tau_{1}$ is long. The mathematically generated curves have been plotted in Figs. 6, 
A

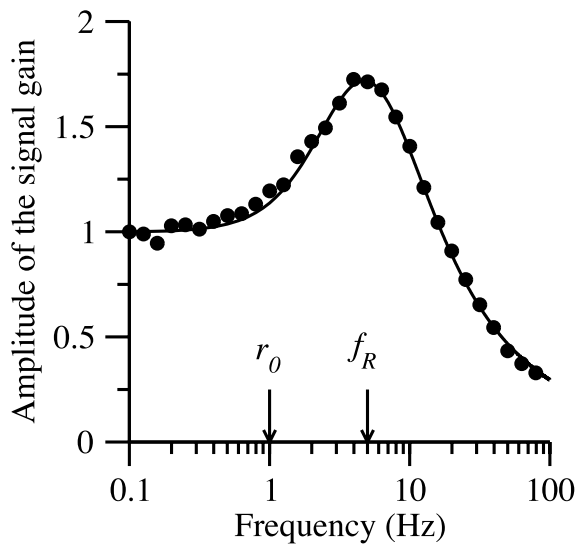

B

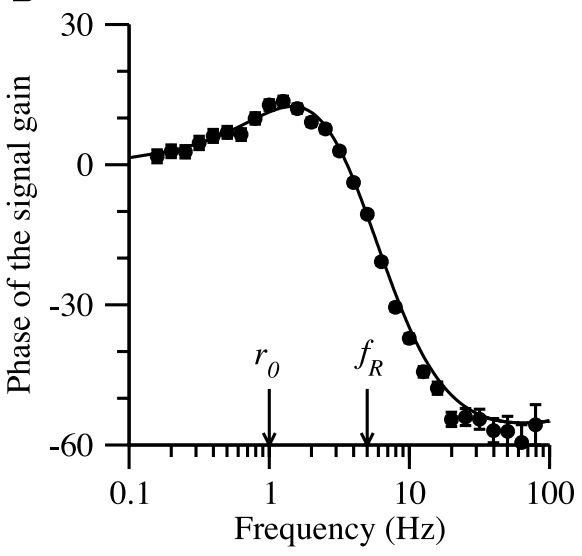

FIG. 7. The signal gain for the case of noisedriven firing at a low rate. GIF model, same parameters as in Fig. 6. $A$ : the amplitude $|A(f)|$. The frequency most strongly amplified is at the subthreshold resonance of $5 \mathrm{~Hz}$. B: the phase $\phi(f)$ of the signal gain. There is a phase advance at low frequencies as well as peak at $\sim 2 \mathrm{~Hz}$. The signal gain curves have been normalized to pass through 1 at $f=0$. - , the theoretical predictions; $\bullet$, from numerical simulations of the model. The firing rate $r_{0}$ and the subthreshold resonance frequency $f_{\mathrm{R}}$ are shown $(\downarrow)$.
$C$ and $D$, and $7, A$ and $B$, for comparison with data from numerical simulation. All signal-gain amplitude profiles are normalized to 1 at $f=0$, see APPENDIX.

The results can be summarized by the following two statements: 1) when the noise is low and the firing regular, a resonant neuron preferentially amplifies input frequencies at the firing rate itself. 2) In cases of irregular noise-driven firing, a resonant neuron will preferentially amplify an input frequency close to its subthreshold resonance. It should be noted that the first mode of amplification is not restricted to resonant neurons. In fact this type of behavior is also seen in passive neurons and has been demonstrated experimentally (Knight 1972). In this situation, the neuron does not directly communicate any information about its subthreshold properties to the network. The second mode of amplification, in which the preferential amplification is at a frequency intrinsic to the cell, is particular to resonant neurons: the mathematical analysis directly links this firing-rate resonance with the existence of a subthreshold resonance.

\section{Conductance-based models}

The analysis of a resonant GIF neuron in the preceding text demonstrated that there are two distinct modes of behavior with respect to the amplification of particular frequencies. In the case of low noise, the frequency most strongly amplified was at the firing rate (a property also of passive neurons), whereas in the case of high noise the amplification was most strong at the intrinsic resonant frequency of the cell.

These results were obtained using the idealized GIF model in which the Hodgkin-Huxley action potential is replaced with a voltage threshold and reset. A further assumption is that the subthreshold behavior is the same at all holding voltages. Another simplification is that a current-based white-noise term was used to model the synaptic bombardment rather than a more realistic temporally correlated conductance-based noise. Given these approximations, it should be tested if the results of the previous section carry over to more complete models that include spike-generating and other voltage-dependent currents together with conductance noise (Destexhe et al. 2001). The preceding analysis was therefore repeated for the two conductance-based model neurons that are described in METHODS and the APPENDIX and for which the subthreshold resonance behaviors are depicted in Figs. 3 and 4.
MODEL I. A NEURON WITH $I_{\mathrm{H}}, I_{\mathrm{NA}}$, AND $I_{\mathrm{K}}$ CURRENTS. The amplitude of the signal gain was examined in both the low- and high-noise regimes analogous to that shown in Figs. 6 and 7. As can be seen in Fig. $4 A$, the subthreshold resonance frequency varies with holding voltage. However, as seen in the $Q$ values in the same figure, the dominating resonance frequency $f_{R}$ is near $10 \mathrm{~Hz}$.

Low noise with high firing rate $r_{O}>f_{\mathrm{R}}$. The synaptic drive was parameterized by the conductances $g_{\text {eo }}=0.01 \mu \mathrm{S}, g_{\text {io }}=$ $0.0085 \mu \mathrm{S}$ and noise strengths $\sigma_{\mathrm{e}}=\sigma_{\mathrm{i}}=0.0005 \mu \mathrm{S}$ eliciting regular firing at a rate $r_{0}$ close to $50 \mathrm{~Hz}$. As can be seen in Fig. $8 A$, the signal gain peaks at this firing-rate frequency.

High noise with high firing rate $r_{O}>f_{\mathrm{R}}$. The synaptic drive was parameterized by the conductances $g_{\text {eo }}=0.01 \mu \mathrm{S}, g_{\text {io }}=$ $0.015 \mu \mathrm{S}$ and noise strengths $\sigma_{\mathrm{e}}=\sigma_{\mathrm{i}}=0.01 \mu \mathrm{S}$ also giving a firing rate $r_{0}$ close to $50 \mathrm{~Hz}$. As expected in this case of irregular firing, the peak in the signal gain (Fig. $8 B$ ) is now at the resonant frequency $f_{\mathrm{R}}=10 \mathrm{~Hz}$.

High noise and low firing rate $r_{O}<f_{\mathrm{R}}$. The synaptic drive was parameterized by the conductances $g_{\text {eo }}=0.01 \mu \mathrm{S}, g_{\text {io }}=$ $0.063 \mu \mathrm{S}$ and noise strengths $\sigma_{\mathrm{e}}=\sigma_{\mathrm{i}}=0.015 \mu \mathrm{S}$ giving a low (but noise-driven) firing rate of $r_{0} \sim 5.5 \mathrm{~Hz}$. As in the previous example of noise-driven firing, the peak in the signal gain is near the resonant frequency of $10 \mathrm{~Hz}$, Fig. $8 C$.

MODEL II. A NEURON WITH $I_{\mathrm{KS}}, I_{\mathrm{NAP}}, I_{\mathrm{NA}}$, AND $I_{\mathrm{K}}$ CURRENTS. The $Q$ values for this model (see Fig. $4 B$ ) demonstrate that the resonance is strong for a range of frequencies with a peak at the onset of spontaneous oscillations near $55 \mathrm{~Hz}$. As the subthreshold resonance is at relatively high frequencies, the only case examined for model II is that for which the firing rate is less than the resonant frequency $r_{0}<f_{\mathrm{R}}$.

Noise-driven firing with $r_{O}<f_{R}$. The synaptic drive was parameterized by the conductances $g_{\text {eo }}=0.02 \mu \mathrm{S}, g_{\text {io }}=0.012$ $\mu \mathrm{S}$ and noise strengths $\sigma_{\mathrm{e}}=\sigma_{\mathrm{i}}=0.01 \mu \mathrm{S}$ inducing a firing rate of $\sim 20 \mathrm{~Hz}$. As can be seen in Fig. $8 D$, the signal gain profile is peaked at 50-55 Hz: near the subthreshold resonance frequency.

EFFECT OF THE CONDUCTANCE INCREASE FROM THE SYNAPTIC DRIVE. It should be noted that the conductance change caused by the modeled noise (see Eq. 8) has the effect of increasing the value of the subthreshold resonance frequency, relative to that which would be measured in the absence of synaptic drive. In Fig. 8, for the case of model I (case of high noise and high 

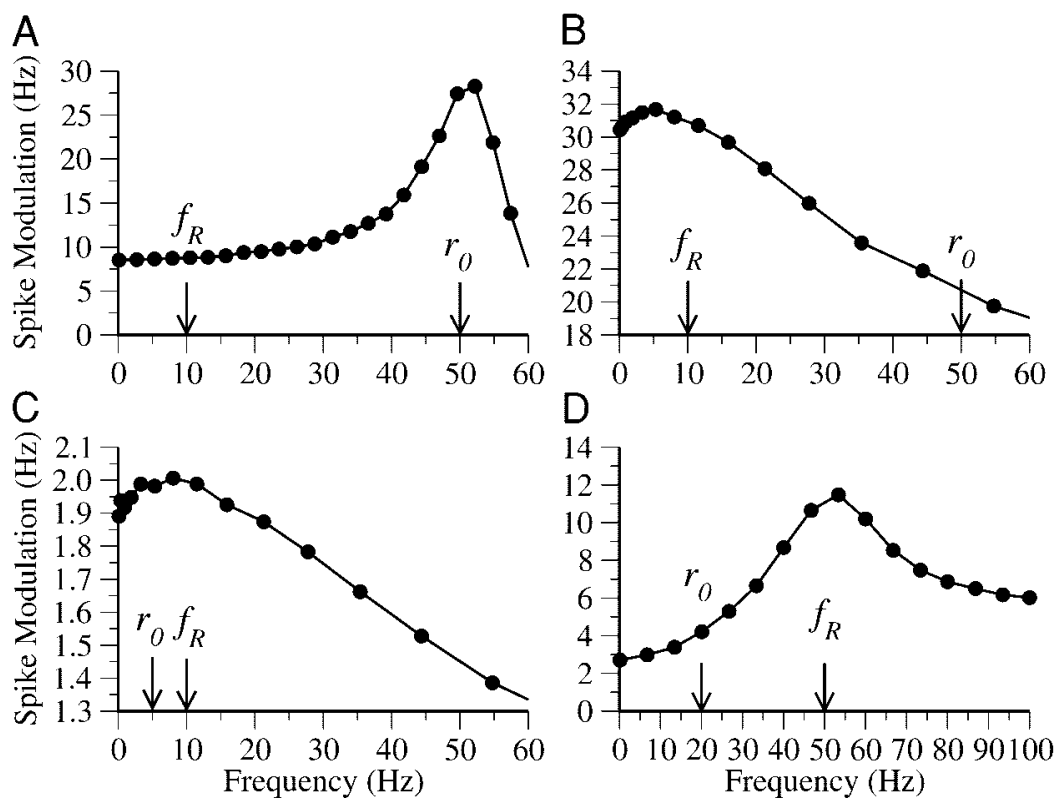

FIG. 8. The spike-response $\left(I_{1}|A(f)|\right)$ to a weak applied oscillating current (amplitude $I_{1}$ ) for regular and noise-driven firing modes for two conductance-based models of resonant neurons. $A$ : model I, regular firing $\left(g_{\text {eо }}=0.01 \mu \mathrm{S}, g_{\text {io }}=0.0085\right.$ $\left.\mu \mathrm{S}, \sigma_{\mathrm{e}}=\sigma_{\mathrm{i}}=0.0005 \mu \mathrm{S}, I_{1}=0.02 \mathrm{nA}\right)$. The strongest amplification is at a frequency equal to the firing rate $r_{0}=50$ Hz. $B$ : model I, noise-driven irregular firing at high rate $\left(g_{\text {eo }}=\right.$ $\left.0.01 \mu \mathrm{S}, g_{\text {io }}=0.015 \mu \mathrm{S}, \sigma_{\mathrm{e}}=\sigma_{\mathrm{i}}=0.01 \mu \mathrm{S}, I_{1}=0.2 \mathrm{nA}\right)$. The strongest amplification is now near the resonant frequency $f_{\mathrm{R}}=$ $10 \mathrm{~Hz}$. $C$ : model I, noise-driven firing at low rate $r_{0} \sim 5.5 \mathrm{~Hz}$ $\left(g_{\text {eo }}=0.01 \mu \mathrm{S}, g_{\text {io }}=0.063 \mu \mathrm{S}, \sigma_{\mathrm{e}}=\sigma_{\mathrm{i}}=0.015 \mu \mathrm{S}, I_{1}=0.1\right.$ $\mathrm{nA})$. Again the peak amplification is at the resonance frequency $f_{\mathrm{R}}=10 \mathrm{~Hz}$. $D$ : model II, noise-driven firing at low rate $\left(g_{\mathrm{eo}}=\right.$ $\left.0.02 \mu \mathrm{S}, g_{\mathrm{io}}=0.012 \mu \mathrm{S}, \sigma_{\mathrm{e}}=\sigma_{\mathrm{i}}=0.01 \mu \mathrm{S}, I_{1}=0.1 \mathrm{nA}\right)$. The strongest amplification is near the resonant frequency of this neuron, at $\sim 50 \mathrm{~Hz}$. The error bars are smaller than the symbol size.

firing rate), the conductance change corresponded to a $70 \%$ increase in the leak. This effect increased the subthreshold resonant frequency by $\sim 1 \mathrm{~Hz}$ over the voltage range -100 to $-60 \mathrm{mV}$. For model II (for which the conductance increase was $90 \%$ ) the corresponding increase in the subthreshold resonant frequency was $<2 \mathrm{~Hz}$. Larger synaptic conductances lead to larger shifts in the subthreshold preferred frequency and consequently in the firing-rate preferred frequency as shown in Fig. 9 for the case of model II. The preferred frequency is close to $50 \mathrm{~Hz}$ for a conductance change of $100 \%$ but increases to $\sim 60$ and $80 \mathrm{~Hz}$ for a conductance change of 200 and $400 \%$ values similar to some estimates of conductance changes in vivo (Destexhe and Paré 1999). The frequency where the spike rate is in phase with the input increases accordingly. The shift of the firing rate resonant frequency is related to the shift in subthreshold frequency. The latter shift can be calculated using $E q$. A6 of the APPENDIX. The strength of subthreshold resonance decreases with an increase in synaptic conductance, near a particular holding voltage. Thus one would expect that the strength of the firing rate resonance would also decrease with the conductance increase. However, this is not necessarily the case, as shown in Fig. 9 where it is seen that the resonance strength increases with the conductance increase. The increase in this case appears to be due to the fact that the neuron spends more time at values of the membrane potential close to threshold, where the subthreshold resonance is stronger (see Fig. 4).

In summary, the conclusions drawn in the analysis of the GIF model neuron also hold for two distinct conductancebased models of resonance: the neurons amplify frequencies near their subthreshold resonance frequency under conditions of noise-driven irregular firing. A supplementary feature is that conductance-based synaptic noise increases the subthreshold resonance frequency. This in turn increases the frequency for which the firing rate is preferentially modulated, in the irregular firing regime.

\section{I S C U S S I O N}

Many classes of neurons throughout the nervous system exhibit subthreshold resonance. In this paper, we systematically studied the effect of this intrinsic frequency preference on both the subthreshold properties and the dynamics of the firing
A

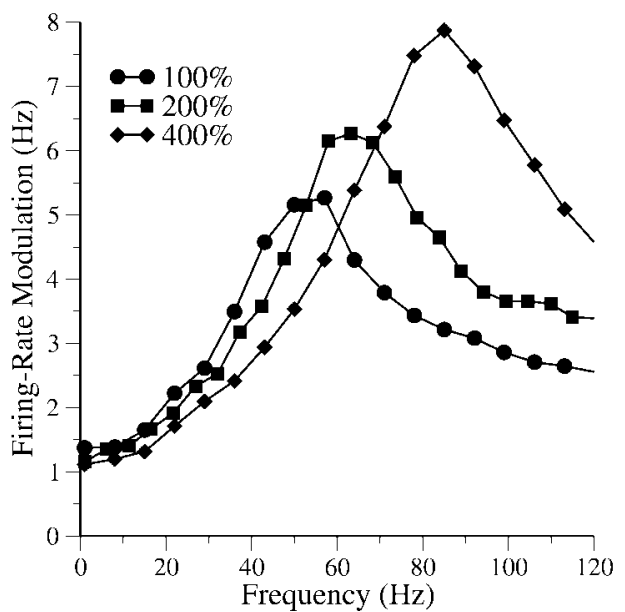

B

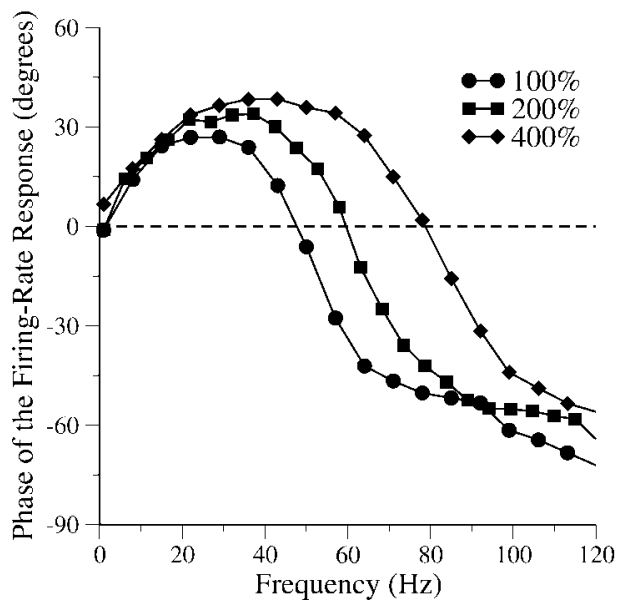

FIG. 9. The effect of increasing synaptic conductance on the modulation and phase of the spike-response $\left(I_{1}|A(f)|\right)$. Model II, for which $g_{\mathrm{L}}=0.037 \mu \mathrm{S}$, with synaptic-conductance input that increases this conductance by $100 \%\left(\bullet, g_{\text {eo }}=0.021 \mu \mathrm{S}, g_{\text {io }}=\right.$ $0.016 \mu \mathrm{S}), 200 \%\left(\mathbf{\square}, g_{\text {eo }}=0.038 \mu \mathrm{S} g_{\mathrm{i}_{\mathrm{o}}}=\right.$ $0.036 \mu \mathrm{S})$, and $400 \%\left(\bullet, g_{\text {eo }}=0.076 \mu \mathrm{S}\right.$, $\left.g_{\text {io }}=0.072 \mu \mathrm{S}\right)$. A: spike rate modulation. The spike-rate resonant frequency is shifted to $\sim 50,60$, and $80 \mathrm{~Hz}$ for conductance increases of 100,200 , and $400 \%$, respectively. $B$ : phase. The frequency where the spike rate is in phase with the input has a corresponding shift to higher values. In all cases, the amplitude of the sinusoidal current was 0.05 $\mathrm{nA}$ and $\sigma_{\mathrm{e}}=\sigma_{\mathrm{i}}=0.01 \mu \mathrm{S}$, eliciting a background firing rate of $20 \mathrm{~Hz}$. 
rate. Using reduced models as well as two example conductance-based model neurons, it was found that resonant neurons can amplify an oscillatory component in their input as they transmit it to postsynaptic targets. When the noise from the synaptic bombardment is significant, the frequency most strongly amplified is the subthreshold resonant frequency itself. The results of this study underline the importance of taking noise into account when examining neuronal response and provides a first step in the understanding of the role of resonance in oscillations at the network level.

\section{Subthreshold dynamics}

Our analysis of two- and three-variable neuronal models classifies a broad range of subthreshold behavior, providing a unifying framework in which different models can be inserted and compared. In particular, it shows that resonance and damped oscillations in response to transient inputs occur in overlapping but different regions of parameters.

Neurons can exhibit resonance when they include a sufficiently slow variable that opposes voltage change as previously emphasized (see e.g., Hutcheon and Yarom 2000). In this case, the negative feedback at low frequencies together with the suppressed response at high frequencies creates a band-pass filter that gives the resonant response but not a damped-oscillatory response. Figure 2 shows that having a sufficiently slow variable that opposes voltage change is indeed a sufficient condition for resonant behavior. It is, however, not a necessary condition because even a fast variable (i.e., $\tau_{1}<C / g$ ) can produce resonance as long as its conductance $g_{1}$ is sufficiently large. In fact, an examination of the regions in Fig. 2 shows that resonant neurons with slow variables are generally nonoscillating, whereas those with fast variables are generally intrinsically oscillating. Conversely, neurons exhibiting damped oscillations do not necessarily have resonance if the damping of the oscillation is strong enough. This appears in a small parameter region where the effective coupling between the two variables is weak, as shown in Fig. 2.

Our analysis also clarifies the relationship between the phase and the amplitude of the impedance. Phase advance of the membrane potential at low frequencies implies a peak in the impedance profile, but the reverse is not necessarily true, if the resonance is too weak.

Further analysis of three-variable models identified an impedance profile with a trough at low frequency followed by a peak at higher frequencies. Such behavior was recently found experimentally in CA1 interneurons of the hippocampus by Pike et al. (2000). The criteria for a trough in the impedance profile are two active components that act on a slower time scale than the membrane time constant. The slowest of the two variables must act to amplify voltage change, whereas the faster should act to oppose the change. An explicit example was given in terms of a neuron with an inactivating slow potassium current.

The analytical results presented can be used in the interpretation of experiment in two ways. They allow for the systematic building of a minimal model of a neuron, given the experimentally measured impedance profile. First the qualitative features of the resonance curve indicate the minimal number of variables needed (e.g., two variables for neurons with a single peak in the curve, three variables for neurons with a trough followed by a peak); second, the parameters of the model can be obtained quantitatively by fitting the impedance curve of the given model to the data. From an experimental point of view, they provide hints for the type of currents, or combination of currents, needed to achieve given resonance properties (see also Hutcheon and Yarom 2000).

The general subthreshold "phase diagrams" that were introduced here are related to several diagrams, which appeared previously in the literature for particular neurons. In models with persistent sodium and slow potassium currents, diagrams have been drawn in parameter space $g_{\mathrm{NaP}^{-}} g_{\mathrm{Ks}}$ (Hutcheon and Yarom 2000; White et al. 1995). In Fig. 2, $\alpha$ is proportional to minus $g_{\mathrm{NaP}}$, whereas $\beta$ is proportional to $g_{\mathrm{Ks}}$. The triangular region (of the above-mentioned diagram) where subthreshold oscillations occur corresponds to the area bounded by the line where spontaneous oscillations arise and the continuation of the straight line indicating a zero total conductance in Fig. 2. Likewise, the diagram of Manor et al. (1997), showing how the cellular behavior changes when the leak conductance and the conductance of a low-threshold calcium current are changed, is related to Fig. 2, by an appropriate change of variables. The conductance of the low-threshold calcium channels enters both on the $\alpha$ variable (through its instantaneous activation dynamics) and on the $\beta$ variable.

\section{Firing-rate dynamics}

Resonance properties have traditionally been studied at the single-neuron level or in neurons coupled by gap junctions (Manor et al. 1997). On the other hand, firing properties of resonant neurons have been less systematically studied. Such studies are important to assess the role of resonance in networks in which the main coupling is chemical and not electrical. Indeed, for subthreshold resonant properties to affect network dynamics, as reported by Tiesinga et al. (2001) and Wang (2002), the firing properties of single neurons themselves should be sensitive to the resonance. In the present paper, this question has been investigated, using both reduced models and conductance-based models. It has been shown how noise plays a crucial role in the appearance of resonance at subthreshold frequencies of the firing rate modulated by a sinusoidal input. When noise is weak and firing is regular, the firing rate response is dominated by resonances at the background firing frequency and the subthreshold resonance is masked. When noise is strong and firing is approximately Poisson, the resonance at the background firing rate disappears and the subthreshold resonance is revealed. The phenomenon is most clearly seen with the GIF model that has a subthreshold response independent of membrane potential and is submitted to white-noise current inputs. This produces a single voltageindependent resonant frequency that can be directly compared with the frequency amplified in the firing rate. Given the simplicity of the model, the shift of the firing rate resonance with noise and its relation to the subthreshold can also be demonstrated mathematically. However, neurons are more realistically modeled using a conductance-based description. In addition, synaptic "noise" is better modeled by conductance fluctuations that both modify the conductance of the neuron and give rise to correlated noise. This complicates the phenomenon as compared with the GIF model. The neuronal conductance and the preferred subthreshold frequency vary with the 
membrane potential and with the input strengths. The effect of increasing synaptic conductance is to increase the subthreshold resonance frequency, and this effect leads, for noisy inputs, to a corresponding increase in firing rate resonance frequency. The noise correlations also modify the neuronal response at high frequency as previously described (Brunel et al. 2001), but this happens at higher frequencies than those of interest here. So the phenomenon observed with the GIF model is found to remain true for the conductance-based models. At a general level of explanation, it can be attributed to the bandpass filter properties of the membrane subthreshold dynamics that amplify the response at the subthreshold preferred frequency.

Our study suggests a functional role for noise in making known to the network the frequency preferences of single neurons. In a sense, noise allows the neurons to communicate to each other information about the particular subthreshold ion-channel dynamics they exhibit, a message that otherwise would be lost in regular spiking dynamics. These results on the firing rate dynamics of resonant neurons could be tested in any slice preparation in which resonant neurons are observed. Between 1,000 and 50,000 s of simulation time were needed to produce accurate numerical data for this paper. However, simulations also suggest that collecting spikes for $\sim 5-10$ min per frequency should be sufficient to see the effects of subthreshold resonance in the signal gain at a reasonable significance level.

It should perhaps be emphasized that the unmasking of subthreshold resonance properties by noise is quite distinct from the usual "stochastic resonance" effect (Wiesenfeld and Moss 1995) that quantifies the output at a given frequency as the strength of noise is varied. In this case, resonance generally arises as some stochastic rate coincides with the given oscillating frequency. A simple IF model, with passive membrane properties, displays stochastic resonance (Bulsara et al. 1994) but not the subthreshold and firing-rate resonance studied here.

Firing rate resonance and the associated phase advance of the firing rate at low frequencies can also be due to other mechanisms, such as spike frequency adaptation (Fuhrmann et al. 2002). One difference between the two mechanisms is that with spike frequency adaptation the negative feedback comes through action potential-dependent currents, whereas in the case of subthreshold resonance negative feedback comes from the interspike subthreshold dynamics. Another difference is that for the case of subthreshold resonance, the peak response in the signal gain and corresponding zero phase lag near the resonant frequency do not appear under noiseless conditions.

\section{Implications for network synchronization}

The firing rate signal gain examined in this paper is one of the crucial quantities that determines the synchronization properties of large networks, together with the temporal characteristics of synaptic transmission. In particular, the existence of phase advance of the firing rate at low frequency can generate an oscillation in networks of excitatory neurons as was shown for the case of adapting neurons (Fuhrmann et al. 2002). Therefore we expect that in noisy in-vivo-like conditions, networks of excitatory neurons with subthreshold resonance could generate oscillations.

From the theoretical point of view, we have introduced a simplified neuronal model for a resonant neuron, the generalized IF neuron, that can be engineered to capture the subthreshold dynamics of any conductance-based model neuron but with a fixed threshold for firing. Simple one-variable IF neurons have proven very useful for understanding the dynamics of large networks because analytical tools can be applied to study and understand in great detail the collective dynamics of such networks (see e.g., Abbott and van Vreeswijk 1993; Amit and Brunel 1997; Amit and Tsodyks 1991; Brunel 2000; Brunel and Hakim 1999; Gerstner 2000; Treves 1993). However, IF neurons are clearly not suited to describe neurons with subthreshold resonance. The generalized IF neuron that was introduced here provides an appropriate tool for the investigation of the collective properties of networks of resonant neurons. Such theoretical studies will help to obtain a deeper understanding of the functional role of subthreshold resonance in the nervous system.

\section{A P P E N D I X}

\section{Model I: spiking neuron with an $I_{H}$ current}

The model is defined as follows

$$
C_{\mathrm{M}} \frac{\mathrm{d} V}{\mathrm{~d} t}=-I_{\mathrm{L}}-I_{\mathrm{H}}-I_{\mathrm{Na}}-I_{\mathrm{K}}-I_{\mathrm{syn}}+I_{\text {app }}
$$

where the membrane capacitance $C_{M}=0.37 \mathrm{nF}$ and the leak current $I_{L}$ is defined by $I_{\mathrm{L}}=\bar{g}_{\mathrm{L}}\left(V-E_{\mathrm{L}}\right)$ with $\bar{g}_{\mathrm{L}}=0.037 \mu \mathrm{S}$ and $E_{\mathrm{L}}=-68$ $\mathrm{mV}$. The other currents take the form

$$
\begin{gathered}
I_{\mathrm{Na}}=\bar{g}_{\mathrm{Na}} m_{\infty}^{3} h\left(V-E_{\mathrm{Na}}\right) \\
I_{\mathrm{K}}=\bar{g}_{\mathrm{K}} n^{4}\left(V-E_{\mathrm{K}}\right) \\
I_{\mathrm{H}}=\bar{g}_{\mathrm{H}}(0.8 f+0.2 s)\left(V-E_{\mathrm{H}}\right)
\end{gathered}
$$

where the conductances $\bar{g}$ and reversal potentials $E$ are given in Table $\mathrm{A} 1$, and it should be noted that $f_{\infty}=s_{\infty}$. The time-varying activation and inactivation variables $h, n, f$, and $s$ all follow equations of the form

TABLE 1. Definitions and parameters of the currents used for the conductance-based models

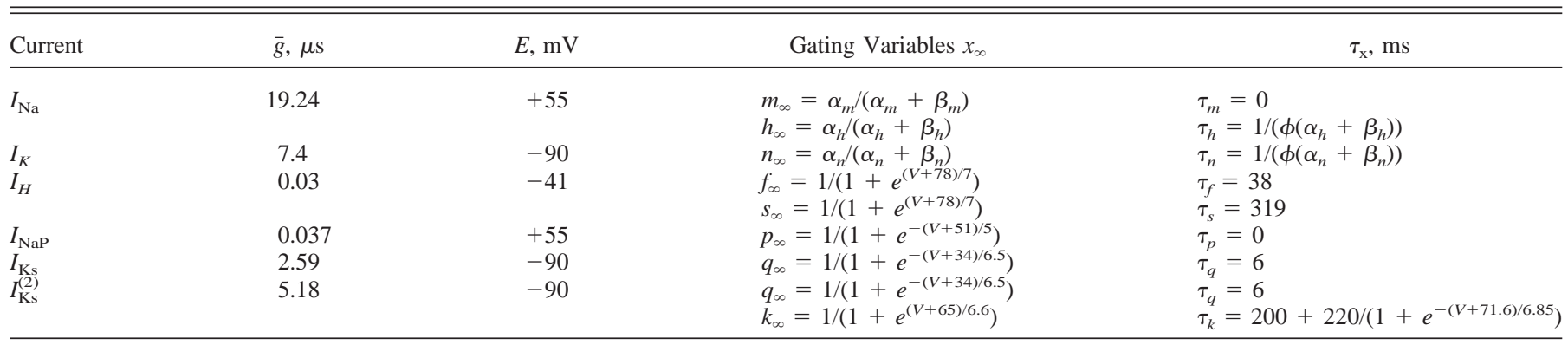


TABLE 2. Values for $\alpha$ and $\beta$ used in the definition of the spikegenerating currents $I_{N a}$ and $I_{k}$

\begin{tabular}{ccc}
\hline \hline & $\alpha$ & $\beta$ \\
\hline$m$ & $\alpha_{m}=\frac{-0.1(V+32)}{\exp (-0.1(V+32))-1}$ & $\beta_{m}=4 \exp (-(V+57) / 18)$ \\
$h$ & $\alpha_{h}=0.07 \exp (-(V+46) / 20)$ & $\beta_{h}=\frac{1}{\exp (-0.1(V+16))+1}$ \\
$n$ & $\alpha_{n}=\frac{-0.01(V+36)}{\exp (-0.1(V+36))-1}$ & $\beta_{n}=0.125 \exp (-(V+46) / 80)$ \\
\hline
\end{tabular}

$$
\tau_{\mathrm{x}}(V) \frac{\mathrm{d} x}{\mathrm{~d} t}=x_{\infty}(V)-x
$$

The voltage dependence of the corresponding $\tau_{x}(V)$ and $x_{\infty}(V)$ parameters are also given in Table A1. All currents are taken to be at physiological temperatures, and hence the time scale of the HodgkinHuxley spike-generating currents have been shortened by the temperature factor $\phi=3^{(36-6.3) / 10}=26.12$. The resting voltage of this model neuron is equal to $V_{\text {rest }}=-65.2 \mathrm{mV}$ and the firing threshold is close to $-56.4 \mathrm{mV}$.

LINEARIZATION. Following the procedure outlined in METHODS, the conductance-based model outlined in the preceding text can be linearized around a holding voltage $V^{*}$ (which can be varied by changing $\left.I_{\text {app }}\right)$. This model yields the following set of linear equations

$$
\begin{aligned}
& C_{\mathrm{M}} \dot{v}=-g_{\mathrm{M}} v-g_{h} w_{h}-g_{n} w_{n}-g_{f} w_{f}-g_{s} w_{s} \\
& \tau_{h} \dot{w}_{h}=v-w_{h} \\
& \tau_{n} \dot{w}_{n}=v-w_{n} \\
& \tau_{f} \dot{w}_{f}=v-w_{f} \\
& \tau_{s} \dot{w}_{s}=v-w_{s}
\end{aligned}
$$

The conductance $g_{\mathrm{M}}=\partial I_{\mathrm{mem}} / \partial V$ is the slope of the instantaneous $I-V$ curve at $V^{*}$

$g_{\mathrm{M}}=\bar{g}_{\mathrm{L}}+\bar{g}_{\mathrm{Na}} m_{\infty}^{3} h_{\infty}+\bar{g}_{\mathrm{K}} n_{\infty}^{4}+\bar{g}_{\mathrm{H}}\left(0.8 f_{\infty}+0.2 s_{\infty}\right)$

$$
+3 \bar{g}_{\mathrm{Na}} m_{\infty}^{2} h_{\infty}\left(V^{*}-E_{\mathrm{Na}}\right)\left(\frac{\partial m_{\infty}}{\partial v}\right)^{*}
$$

where the convention that $X^{*}$ is the quantity $X$ evaluated at the holding voltage $V^{*}$ is used. The other conductances introduced are

$$
\begin{gathered}
g_{\mathrm{h}}=\bar{g}_{\mathrm{Na}}\left(V^{*}-E_{\mathrm{Na}}\right) m_{\infty}^{3}\left(\frac{\delta h_{\infty}}{\delta v}\right)^{*} \\
g_{\mathrm{n}}=4 \bar{g}_{\mathrm{K}}\left(V^{*}-E_{\mathrm{K}}\right) n_{\infty}^{3}\left(\frac{\delta n_{\infty}}{\delta v}\right)^{*} \\
g_{\mathrm{f}}=\bar{g}_{\mathrm{H}} 0.8\left(V^{*}-E_{\mathrm{H}}\right)\left(\frac{\delta f_{\infty}}{\delta v}\right)^{*} \\
g_{\mathrm{s}}=\bar{g}_{\mathrm{H}} 0.2\left(V^{*}-E_{\mathrm{H}}\right)\left(\frac{\delta s_{\infty}}{\delta v}\right)^{*}
\end{gathered}
$$

REDUCTION TO TWO VARIABLES. The variables $w_{h}$ and $w_{n}$ are much faster than other time scales in the system. Thus the substitution $w_{h}=v$ and $w_{n}=v$ in the voltage equation represents a good approximation. The slow variable of the $H$ current $w_{s}$ has a relatively long time scale and hence follows the time average of $v$ (obtained from a solution of $E q$. Al). For voltage changes that average to zero around the rest, the replacement $w_{s} \rightarrow 0$ is a valid approximation. These approximations taken together yield the two-variable system of equations

$$
C \frac{\mathrm{d} v}{\mathrm{~d} t}=-g v-g_{f} w_{f}
$$

$$
\tau_{f} \frac{\mathrm{d} w_{f}}{d t}=v-w_{f}
$$

where $C=C_{\mathrm{M}}$ and $g=g_{\mathrm{M}}+g_{h}+g_{n}$.

\section{Model II}

The model is defined as follows

$$
C_{\mathrm{M}} \frac{\mathrm{d} V}{\mathrm{~d} t}=-I_{\mathrm{L}}-I_{\mathrm{NaP}}-I_{\mathrm{Ks}}-I_{\mathrm{Na}}-I_{\mathrm{K}}-I_{\mathrm{syn}}+I_{\mathrm{app}}
$$

where $C_{\mathrm{M}}=0.37 \mathrm{nF}$ and the leak current is defined by $I_{\mathrm{L}}=\bar{g}_{\mathrm{L}}(V-$ $E_{\mathrm{L}}$ ) with $\bar{g}_{\mathrm{L}}=0.037 \mu \mathrm{S}$ and $E_{\mathrm{L}}=-60 \mathrm{mV}$. The spike-generating currents are identical to those of model I. The $I_{\mathrm{NaP}}$ and noninactivating $I_{\mathrm{Ks}}$ currents are defined (Wang 1993) by

$$
\begin{gathered}
I_{\mathrm{NaP}}=\bar{g}_{\mathrm{NaP}} p_{\infty}\left(V-E_{\mathrm{NaP}}\right) \\
I_{\mathrm{Ks}}=\bar{g}_{\mathrm{Ks}} q\left(V-E_{\mathrm{Ks}}\right)
\end{gathered}
$$

The resting voltage of the neuron is $V_{\text {rest }}=-66.2 \mathrm{mV}$ and the onset of spontaneous oscillations occurs near $-57.2 \mathrm{mV}$.

LINEARIZATION. Linearizing this model at a fixed voltage $V^{*}$ yields the following set of linear differential equations

$$
\begin{gathered}
C \dot{v}=-g_{\mathrm{M}} v-g_{h} w_{h}-g_{n} w_{n}-g_{q} w_{q} \\
\tau_{h} \dot{w}_{h}=v-w_{h} \\
\tau_{n} \dot{w}_{n}=v-w_{n} \\
\tau_{q} \dot{w}_{q}=v-w_{q}
\end{gathered}
$$

where again the conductance $g_{\mathrm{M}}=\partial I_{\text {mem }} / \mathrm{d} V$ is the slope of the instantaneous $I-V$ curve at $V^{*}$

$g_{\mathrm{M}}=\bar{g}_{\mathrm{L}}+\bar{g}_{\mathrm{Na}} m_{\infty}^{3} h_{\infty}+\bar{g}_{\mathrm{K}} n_{\infty}^{4}+\bar{g}_{\mathrm{NaP}} p_{\infty}+\bar{g}_{\mathrm{Ks}} q_{\infty}+3 \bar{g}_{\mathrm{Na}} m_{\infty}^{2} h_{\infty}\left(V^{*}-E_{\mathrm{Na}}\right)\left(\frac{\partial m_{\infty}}{\partial v}\right)$

$$
+\bar{g}_{\mathrm{NaP}}\left(V^{*}-E_{\mathrm{Na}}\right)\left(\frac{\partial p_{\infty}}{\partial v}\right)^{*}
$$

and the other conductance not common to model I is

$$
g_{\mathrm{q}}=\bar{g}_{\mathrm{Ks}}\left(V^{*}-E_{\mathrm{K}}\right)\left(\frac{\mathrm{d} q_{\infty}}{\mathrm{d} v}\right)^{*}
$$

where all voltage-dependent quantities are evaluated at $V^{*}$.

REDUCTION TO TWO VARIABLES. Again the model can be reduced to the two-variable form by noting that the time scales of the spikegenerating currents are much faster than other time scales in the voltage equation. However, in this case the replacements $w_{h}=v-$ $g_{h} \tau_{h} \dot{v}$ and $w_{n}=v-g_{n} \tau_{n} \dot{v}$ are used, giving a better agreement near the line where spontaneous oscillations appear. Thus the two-variable reduction of this model is

$$
\begin{gathered}
C \frac{\mathrm{d} v}{\mathrm{~d} t}=-v g-g_{q} w_{q} \\
\tau_{q} \frac{\mathrm{d} w_{q}}{\mathrm{~d} t}=v-w_{q}
\end{gathered}
$$

where $C=C_{\mathrm{M}}-g_{h} \tau_{h}-g_{n} \tau_{n}$ and $g=g_{\mathrm{M}}+g_{h}+g_{n}$. 


\section{Model with a trough in the impedance}

This model is similar to model II defined in the preceding text, except that in this case an inactivation term is included in the definition of the slow potassium current

$$
I_{\mathrm{Ks}}^{(2)}=\bar{g}_{\mathrm{Ks}} q k\left(V-E_{\mathrm{K}}\right)
$$

see Table A1 for the values of the various parameters. This model is linearized around $-65 \mathrm{mV}$ to give the following set of equations

$$
\begin{aligned}
C \dot{v}=-g_{M} v-g_{h} w_{h} & -g_{n} w_{n}-g_{q} w_{q}-g_{k} w_{k} \\
\tau_{h} \dot{w}_{h} & =v-w_{h} \\
\tau_{n} \dot{w}_{n} & =v-w_{n} \\
\tau_{q} \dot{w}_{q} & =v-w_{q} \\
\tau_{k} \dot{w}_{k} & =v-w_{k}
\end{aligned}
$$

where in this case

$$
\begin{aligned}
g_{\mathrm{M}}=\bar{g}_{\mathrm{Na}} m_{\infty}^{3} h_{\infty}+\bar{g}_{\mathrm{K}} n_{\infty}^{4}+\bar{g}_{\mathrm{Na}} p_{\infty}+\bar{g}_{\mathrm{Ks}} q_{\infty} k_{\infty}+\bar{g}_{\mathrm{NaP}}\left(V^{*}-E_{\mathrm{Na}}\right)\left(\frac{\partial p_{\infty}}{\partial v}\right)^{*} \\
+3 \bar{g}_{\mathrm{Na}} m_{\infty}^{2} h_{\infty}\left(V^{*}-E_{\mathrm{Na}}\right)\left(\frac{\partial m_{\infty}}{\partial v}\right)^{*}
\end{aligned}
$$

and

$$
\begin{aligned}
& g_{k}=\bar{g}_{\mathrm{Ks}}\left(V^{*}-E_{\mathrm{K}}\right) q_{\infty}\left(\frac{\partial k_{\infty}}{\partial v}\right)^{*} \\
& g_{q}=\bar{g}_{\mathrm{Ks}}\left(V^{*}-E_{\mathrm{K}}\right) k_{\infty}\left(\frac{\partial q_{\infty}}{\partial v}\right)^{*}
\end{aligned}
$$

The corresponding three-variable model is found by assuming the $h$ and $n$ variables are fast, leaving the set

$$
\begin{gathered}
C \dot{v}=-g v-g_{q} w_{q}-g_{k} w_{k} \\
\tau_{q} \dot{w}_{q}=v-w_{q} \\
\tau_{k} \dot{w}_{k}=v-w_{k}
\end{gathered}
$$

where $C=C_{\mathrm{M}}-g_{h} \tau_{h}-h_{n} \tau_{n}$ and $g_{\mathrm{M}}=g+g_{n}+g_{h}$.

The two-variable approximation of this model is obtained by the further assumption that the $k$ variable is slow, and hence $w_{k}$ is set to zero. At $V^{*}=-65 \mathrm{mV}$, this two-variable model is identical to that of model II given in the preceding text. The impedance curves of the full, three-, and two-variables models are given in Fig. $3 B$.

\section{Impedance and eigenvalues of the two-variable model}

The eigenvalues of the two-variable model are

$$
\begin{aligned}
& \lambda_{1}=\frac{1}{2 \tau_{1}}\left(-(\alpha+1)+\sqrt{(\alpha-1)^{2}-4 \beta}\right) \\
& \lambda_{2}=\frac{1}{2 \tau_{1}}\left(-(\alpha+1)-\sqrt{(\alpha-1)^{2}-4 \beta}\right)
\end{aligned}
$$

STABILITY OF THE MEMBRANE POTENTIAL. For the membrane potential to be stable, the real part of both eigenvalues must be negative. This gives the following bounds on the parameters $\alpha$ and $\beta$

$$
\alpha>-1 \text { and } \alpha+\beta>0
$$

AMPLITUDE OF THE IMPEDANCE. For an oscillating current of frequency $f=\omega /(2 \pi)$, the amplitude of the complex impedance $|Z|$ is given as

$$
|Z|=\left(\frac{\tau_{1}}{C}\right) \sqrt{\frac{\left(1+\tau_{1}^{2} \omega^{2}\right)}{\left(\alpha+\beta-\tau_{1}^{2} \omega^{2}\right)^{2}+\tau_{1}^{2} \omega^{2}(1+\alpha)^{2}}}
$$

A subthreshold resonance, signaled by the existence of peak in $|Z(f)|$ at some nonzero frequency, occurs if (Hutcheon et al. 1996a)

$$
\beta>\sqrt{(\alpha+1)^{2}+1}-(1+\alpha)
$$

and at a frequency

$$
\left.f_{\mathrm{R}}=\frac{1}{2 \pi \tau_{1}}\left((\alpha+\beta+1)^{2}-(\alpha+1)^{2}\right)^{1 / 2}-1\right)^{1 / 2}
$$

THE PHASE OF THE IMPEDANCE. The phase $\theta$, defined in $E q .11$, is

$$
\tan \theta=\tau_{1} \omega \frac{\beta-\left(1+\tau_{1}^{2} \omega^{2}\right)}{\beta+\alpha\left(1+\tau_{1}^{2} \omega^{2}\right)}
$$

A zero phase-lag exists for $\beta>1$, whereas a phase lag $>90^{\circ}$ is present for $\alpha<0$.

RESPONSE TO A SQUARE-PULSE CURRENT. The response of the neuron to the onset of a square-pulse current $I_{\text {app }}=I \Theta(t)$ can be found in terms of the eigenvalues as

$$
v=v_{\infty}+v_{\infty}\left(e^{\lambda_{1} t}\left(\frac{\tau_{1} \lambda_{1} \lambda_{2}+\lambda_{2}}{\lambda_{1}-\lambda_{2}}\right)+e^{\lambda_{2} t}\left(\frac{\tau_{1} \lambda_{1} \lambda_{2}+\lambda_{1}}{\lambda_{2}-\lambda_{1}}\right)\right)
$$

where $v_{\infty}=I /\left(g+g_{1}\right)$ is the holding voltage corresponding to an applied current of $I_{\text {app }}=I$ in the linear approximation. When the roots are complex and written as $\lambda=-\mu \pm i \omega_{0}$ the form above can be written more conveniently as

$$
v=v_{\infty}-v_{\infty} e^{-\mu t}\left(\cos \left(\omega_{0} t\right)+\left(\frac{\mu-\tau_{1}\left(\mu^{2}+\omega_{0}^{2}\right)}{\omega_{0}}\right) \sin \left(\omega_{0} t\right)\right.
$$

The existence of complex eigenvalues indicates that the neuron has damped oscillations at a frequency $f_{0}$. This occurs when $4 \beta>(\alpha-$ $1)^{2}$ (Puil et al. 1986) giving

$$
\omega_{0}=\frac{1}{2 \tau_{1}} \sqrt{4 \beta-(\alpha+1)^{2}}
$$

The qualitatively different types of behavior that can occur are the following: when $\beta>(\alpha-1)^{2} / 4$, the membrane responds with damped oscillations; when $\alpha>1$ and $0<\beta<(\alpha-1)^{2} / 4$, the membrane has a single overshoot (or sag if the current pulse is hyperpolarizing); for all other cases, $\alpha<1, \beta<(\alpha-1)^{2} / 4$ and $\alpha>$ $1, \beta<0$, the voltage changes monotonically from its initial to final value.

\section{Impedance and eigenvalues of the three-variable model}

The complex impedance of the three-variable model model defined by $E q .7$ is

$Z=\frac{1}{g}\left(\frac{\left(1+i \omega \tau_{1}\right)\left(1+i \omega \tau_{2}\right)}{\left(1+i \omega \tau_{m}\right)\left(1+i \omega \tau_{1}\right)\left(1+i \omega \tau_{2}\right)+\gamma_{1}\left(1+i \omega \tau_{2}\right)+\gamma_{2}\left(1+i \omega \tau_{1}\right)}\right)$

where $\omega=2 \pi f, \tau_{m}=C / g, \gamma_{1}=g_{1} / g$, and $\gamma_{2}=g_{2} / g$.

To classify the behavior of the amplitude of the impedance versus frequency curve in terms of the number of local minima and/or maxima, the derivative of $|Z|$ with respect to $\omega$ is computed. Its sign is determined by a fourth order polynomial in $\omega^{2}$. The number of real positive roots of this polynomial tell us how many local extrema there are in the amplitude versus frequency curve. The roots of the polynomial are found numerically. From these roots, the boundaries of the regions with 0,1 , or 2 local minima, corresponding respectively to no resonance, simple resonance, and resonance preceded by a trough, are found numerically.

The eigenvalues of the stability matrix of Eq. 7 are given by the following cubic polynomial 


$$
\lambda^{3}+\left(\frac{1}{\tau_{m}}+\frac{1}{\tau_{1}}+\frac{1}{\tau_{2}}\right) \lambda^{2}+\left(\frac{1}{\tau_{1} \tau_{2}}+\frac{1+\gamma_{1}}{\tau_{1} \tau_{m}}+\frac{1+\gamma_{2}}{\tau_{2} \tau_{m}}\right) \lambda+\frac{1+\gamma_{1}+\gamma_{2}}{\tau_{m} \tau_{1} \tau_{2}}=0
$$

Insertion of $\lambda=0$ in Eq. A8 yields the line $1+\gamma_{1}+\gamma_{2}=0$. To find the line where spontaneous oscillations appear, we set $\lambda=i \omega$ and obtain a half straight line in a parametric way

$$
\begin{gathered}
\gamma_{1}=\frac{\tau_{m}+\tau_{2}}{\tau_{1}-\tau_{2}}\left(1+\omega^{2} \tau_{1}^{2}\right) \\
\gamma_{2}=-\frac{\tau_{m}+\tau_{1}}{\tau_{1}-\tau_{2}}\left(1+\omega^{2} \tau_{2}^{2}\right)
\end{gathered}
$$

Finally, to obtain the regions where damped oscillatory behavior arise in response to a step current, the roots of the cubic polynomial are computed numerically. The boundaries of the area in which a pair of complex conjugate roots appear are then found.

\section{Calculation of the firing-rate resonance}

The modulation in the instantaneous firing rate due to a sinusoidal input in the limit $\tau_{1} \gg \tau$ can be calculated analytically. Details will be published elsewhere (Brunel et al. 2003). The modulation in the firing rate can be written

$$
\begin{gathered}
A(\omega)=\left(1-\frac{\gamma Y(\omega)}{1+\gamma Y(\omega)+i \omega \tau_{1}}\right) A_{\mathrm{LIF}}(\omega) \\
Y(\omega)=\frac{1-g \tau_{m}\left(v_{\theta}-v_{\mathrm{r}}\right) A_{\mathrm{LIF}}(\omega)}{1+i \omega \tau_{m}}
\end{gathered}
$$

where $\gamma=g_{1} / g, \tau_{m}=C / g$, and $A_{L I F}(\omega)$ is the firing-rate linear response (signal gain) of the IF neuron (Brunel and Hakim 1999; Brunel et al. 2001)

$$
A_{\mathrm{LIF}}(\omega)=\frac{r_{0}}{g \sigma\left(1+i \omega \tau_{m}\right)}\left(\frac{\frac{\partial U}{\partial y}\left(y_{t}, \omega \tau_{m}\right)-\frac{\partial U}{\partial y}\left(y_{r}, \omega \tau_{m}\right)}{U\left(y_{t}, \omega \tau_{m}\right)-U\left(y_{r}, \omega \tau_{m}\right)}\right)
$$

the noise strength here is given by $\sigma=\left(I_{N} / g\right) \sqrt{\tau_{N} / \tau_{m}}$. The parameters $y_{t}, y_{r}$ and the background firing rate $r_{0}$ are given by the set of equations

$$
\begin{gathered}
y_{t}=\frac{v_{\theta}+\gamma\langle v\rangle-I_{0} / g}{\sigma} \\
y_{r}=\frac{v_{\mathrm{r}}+\gamma\langle v\rangle-I_{0} / g}{\sigma} \\
\langle v\rangle=\frac{I_{0} / g-\left(v_{\theta}-v_{r}\right) r_{0} \tau_{m}}{1+\gamma} \\
r_{0}=\left(\tau_{m} \sqrt{\pi} \int_{y_{r}}^{y_{t}} e^{\mathrm{u}^{2}}[1+\operatorname{erf}(u)]\right)^{-1}
\end{gathered}
$$

where $\operatorname{erf}(u)$ is the standard error function, and $U$ is given in terms of combinations of hypergeometric functions (Abramowitz and Stegun 1970)

$U(y, \omega)=\frac{e^{y^{2}}}{\Gamma((1+i \omega) / 2)} M\left(\frac{1-i \omega}{2}, \frac{1}{2},-y^{2}\right)+\frac{2 y e^{y^{2}}}{\Gamma(i \omega / 2)} M\left(1-\frac{i \omega}{2}, \frac{3}{2},-y^{2}\right)$

The amplitudes of the signal-gain profiles $|A(f)|$ in Figs. 6 and 7 have been normalized to 1 at zero frequency for both the analytical results (-) and data from numerical simulation $(\bullet)$. A systematic and frequency-independent shift of $\sim 10 \%$ between theory and numerics was visible in the unnormalized curves due to the finite value of $\tau_{1}$.
Such a shift can be captured analytically by examining higher orders of the approximation that take the finite value of $\tau_{1}$ into account.

We thank O. Paulsen for useful discussions, as well as C. Meunier and A. Tonnelier for detailed comments on a previous version of the manuscript.

M.J.E. Richardson acknowledges support from a European Community Marie Curie Fellowship. Laboratoire de Physique Statistique is Centre National de la Recherche Scientifique Unité Mixte de Recherche 8550, Associé aux Universités Paris VI et VII.

\section{REFERENCES}

Abbott LF, and van Vreeswijk C. Asynchronous states in networks of pulse-coupled oscillators. Phys Rev E 48: 1483-1490, 1993.

Abramowitz M and Stegun IA. Tables of Mathematical Functions. New York: Dover Publications, 1970.

Adrian ED and Matthews BHC. The Berger rhythm: potential changes from the occipital lobes in man. Brain 57: 355-385, 1934.

Amit DJ and Brunel N. Model of global spontaneous activity and local structured activity during delay periods in the cerebral cortex. Cereb Cortex 7: 237-252, 1997.

Amit DJ and Tsodyks MV. Quantitative study of attractor neural network retrieving at low spike rates. I. Substrate spikes, rates and neuronal gain. Network 2: 259-274, 1991.

Anderson JS, Lampl I, Gillespie DC, and Ferster D. The contribution of noise to contrast invariance of orientation tuning in cat visual cortex. Science 290: 1968-1972, 2000.

Brunel N. Dynamics of sparsely connected networks of excitatory and inhibitory spiking neurons. J Comput Neurosci 8: 183-208, 2000.

Brunel N, Chance FS, Fourcaud N, and Abbott LF. Effects of synaptic noise and filtering on the frequency response of spiking neurons. Phys Rev Lett 86: 2186-2189, 2001.

Brunel N and Hakim V. Fast global oscillations in networks of integrateand-fire neurons with low firing rates. Neural Comput 11: 1621-1671, 1999.

Brunel B, Hakim V, and Richardson MJE. Firing rate resonance in a generalized integrate-and-fire neuron with subthreshold resonance. Phys Rev In press.

Bulsara AR, Lowen SB, and Rees CD. Cooperative behavior in the periodically modulated Wiener process: noise-induced complexity in a model neuron. Phys Rev E 49: 4989-5000, 1994.

De Zeeuw CI, Simpson JI, Hoogenraad CC, Galjart N, Koekkoek SK, and Ruigrok TJ. Microcircuitry and function of the inferior olive. Trends Neurosci 21: 391-400, 1998.

Destexhe A and Paré D. Impact of network activity on the integrative properties of neocortical pyramidal neurons in vivo. J Neurophysiol 81: 1531-1547, 1999.

Destexhe A, Rudolph M, Fellous J-M, and Sejnowski TJ. Fluctuating synaptic conductances recreate in vivo-like activity in neocortical neurons. Neuroscience 107: 13-24, 2001.

Dickson CT, Magistretti J, Shalinsky MH, Fransen E, Hasselmo ME, and Alonso A. Properties and role of $I(\mathrm{~h})$ in the pacing of subthreshold oscillations in entorhinal cortex layer II neurons. J Neurophysiol 83: 2562-2579, 2000.

Fuhrmann G, Markram H, and Tsodyks M. Spike frequency adaptation and neocortical rhythms. J Neurophysiol 88: 761-770, 2002.

Gerstner W. Population dynamics of spiking neurons: fast transients, asynchronous states, and locking. Neural Comput 12: 43-89, 2000.

Gutfreund Y, Yarom Y, and Segev I. Subthreshold oscillations and resonant frequency in guinea pig cortical neurons: physiology and modelling. J Physiol 483: 621-640, 1995.

Hodgkin AL and Huxley AF. A quantitative description of membrane current and its application to conductance and excitation in nerve. J Physiol 117: 500-544, 1952.

Honeycutt RL. Stochastic Runge-Kutta algorithms. I. White noise. Phys Rev A 45: 600-603, 1992.

Hutcheon B, Miura RM, and Puil E. Models of subthreshold membrane resonance in neocortical neurons. J Neurophysiol 76: 698-714, 1996a.

Hutcheon B, Miura RM, and Puil E. Subthreshold membrane resonance in neocortical neurons. J Neurophysiol 76: 683-697, 1996b.

Hutcheon B, Miura RM, Yarom Y, and Puil E. Low-threshold calcium current and resonance in thalamic neurons: a model of frequency preference. J Neurophysiol 71: 583-594, 1994.

Hutcheon B and Yarom Y. Resonance, oscillation and the intrinsic frequency preferences of neurons. Trends Neurosci 23: 216-222, 2000. 
Izhikevich EM. Resonate-and-fire neurons. Neural Networks 14: 883-894, 2001.

Jahnsen H and Karnup S. A spectral analysis of the integration of artificial synaptic potentials in mammalian central neurons. Brain Res 666: 9-20, 1994.

Knight BW. The relationship between the firing rate of a single neuron and the level of activity in a population of neurons. J Gen Physiol 59: 767-778, 1972.

Koch C. Cable theory in neurons with active linearized membrane. Biol Cybern 50: 15-33, 1984.

Koch C. Biophysics of Computation: Information Processing in Single Neurons. Oxford, UK: Oxford Univ. Press, 1999.

Lampl I and Yarom Y. Subthreshold oscillations of the membrane potential: a functional synchronizing and timing device. J Neurophysiol 70: 21812186, 1993.

Lampl I and Yarom Y. Subthreshold oscillations and resonant behavior: two manifestations of the same mechanism. Neuroscience 78: 325-341, 1997.

Leung LS and Yu HW. Theta-frequency resonance in hippocampal CA1 neurons in vitro demonstrated by sinusoidal current injection. $J$ Neurophysiol 79: 1592-1596, 1998.

Llinás R, Grace AA, and Yarom Y. In vitro neurons in mammalian cortical layer 4 exhibit intrinsic oscillatory activity in the $10-$ to $50-\mathrm{Hz}$ frequency range. Proc Natl Acad Sci USA 88: 897-901, 1991.

Llinás R and Yarom Y. Oscillatory properties of guinea pig olivary neurons and their pharmacological modulation: an in vitro study. J Physiol 376: 163-182, 1986.

Manor Y, Rinzel J, Segev I, and Yarom Y. Low-amplitude oscillations in the inferior olive: a model based on electrical coupling of neurons with heterogeneous channel densitites. J Neurophysiol 77: 2736-2752, 1997.
Mauro A, Conti F, Dodge F, and Schor R. Subthreshold behavior and phenomenological impedance of the squid giant axon. J Gen Physiol 55: 497-523, 1970.

Pike FG, Goddard RS, Suckling JM, Ganter P, Kasthuri N, and Paulsen O. Distinct frequency preferences of different types of rat hippocampal neurons in response to oscillatory input currents. J Physiol 529: 205-213, 2000.

Puil E, Gimbarzevsky B, and Miura RM. Quantification of membrane properties of trigeminal root ganglions in guinea pigs. J Neurophysiol 55: 995-1016, 1986.

Puil E, Meiri H, and Yarom Y. Resonant behavior and frequency preferences of thalamic neurons. J Neurophysiol 71: 575-582, 1994.

Rinzel J and Ermentrout GB. Analysis of neural excitability and oscillations. In: Methods in Neuronal Modeling. Cambridge, MA: MIT Press, 1989, p. 135-169.

Spain WJ, Schwindt PC, and Crill WE. Anomalous rectification in neurons from cat sensorimotor cortex in vitro. J Neurophysiol 57: 1555-1576, 1987.

Tiesinga PHE, Fellous JM, Jose JV, and Sejnowski TJ. Computational model of carbachol-induced delta, theta, and gamma oscillations in the hippocampus. Hippocampus 11: 251-274, 2001.

Treves A. Mean-field analysis of neuronal spike dynamics. Network 4: 259284, 1993.

Wang XJ. Ionic basis for intrinsic oscillations. Neuroreport 5: 221-224, 1993.

Wang X-J. Pacemaker neurons for the theta rhythm and their synchronization in the septohippocampal reciprocal loop. J Neurophysiol 87: 889-900, 2002

White JA, Budde T, and Kay AR. A bifurcation analysis of neuronal subthreshold oscillations. Biophys J 69: 1203-1217, 1995.

Wiesenfeld $\mathbf{K}$ and Moss F. Stochastic resonance and the benefits of noise: from ice ages to crayfish and squids. Nature 373: 33-36, 1995. 